\title{
Carbon and nitrogen abundances of individual stars in the Sculptor dwarf spheroidal galaxy ${ }^{\star}$
}

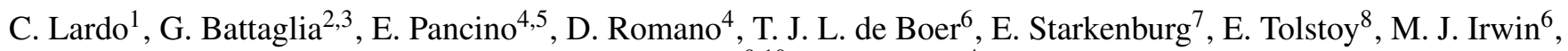 \\ P. Jablonka ${ }^{9,10}$, and M. Tosi ${ }^{4}$
}

\author{
1 Astrophysics Research Institute, Liverpool John Moores University, 146 Brownlow Hill, Liverpool L3 5RF, UK \\ e-mail: C.Lardo@ljmu.ac.uk \\ 2 Instituto de Astrofisica de Canarias, 38205 La Laguna, Tenerife, Spain \\ 3 Universidad de la Laguna, Dpto. Astrofisica, 38206 La Laguna, Tenerife, Spain \\ 4 INAF Osservatorio Astronomico di Bologna, via Ranzani 1, 40127 Bologna, Italy \\ 5 ASI Science Data Center, via del Politecnico SNC, 00133 Roma, Italy \\ 6 Institute of Astronomy, University of Cambridge, Madingley Road, Cambridge CB3 OHA, UK \\ ${ }^{7}$ Leibniz-Institute for Astrophysics Potsdam (AIP), An der Sternwarte 16, 14482 Potsdam, Germany \\ 8 Kapteyn Astronomical Institute, University of Groningen, PO Box 800, 9700AV Groningen, The Netherlands \\ ${ }^{9}$ Laboratoire d' astrophysique, École Polytechnique Fédérale de Lausanne (EPFL), Observatoire, 1290 Versoix, Switzerland \\ 10 GEPI, Observatoire de Paris, CNRS, Université de Paris Diderot, 92195 Meudon, Cedex, France
}

Received 17 September 2015 / Accepted 26 October 2015

\begin{abstract}
We present $[\mathrm{C} / \mathrm{Fe}]$ and $[\mathrm{N} / \mathrm{Fe}]$ abundance ratios and $\mathrm{CH}(\lambda 4300)$ and $\mathrm{S}(\lambda 3883)$ index measurements for 94 red giant branch (RGB) stars in the Sculptor dwarf spheroidal galaxy from VLT/VIMOS MOS observations at a resolving power $R=1150$ at $4020 \AA$. This is the first time that $[\mathrm{N} / \mathrm{Fe}]$ abundances are derived for a large number of stars in a dwarf spheroidal. We found a trend for the [C/Fe] abundance to decrease with increasing luminosity on the RGB across the whole metallicity range, a phenomenon observed in both field and globular cluster giants, which can be interpreted in the framework of evolutionary mixing of partially processed CNO material. Both our measurements of $[\mathrm{C} / \mathrm{Fe}]$ and $[\mathrm{N} / \mathrm{Fe}]$ are in good agreement with the theoretical predictions for stars at similar luminosity and metallicity. We detected a dispersion in the carbon abundance at a given $[\mathrm{Fe} / \mathrm{H}]$, which cannot be ascribed to measurement uncertainties alone. We interpret this observational evidence as the result of the contribution of different nucleosynthesis sources over time to a not well-mixed interstellar medium. We report the discovery of two new carbon-enhanced, metal-poor stars. These are likely the result of pollution from material enriched by asymptotic giant branch stars, as indicated by our estimates of $[\mathrm{Ba} / \mathrm{Fe}]>+1$. We also attempted a search for dissolved globular clusters in the field of the galaxy by looking for the distinctive C-N pattern of second population globular clusters stars in a previously detected, very metal-poor, chemodynamical substructure. We do not detect chemical anomalies among this group of stars. However, small number statistics and limited spatial coverage do not allow us to exclude the hypotheses that this substructure forms part of a tidally shredded globular cluster.
\end{abstract}

Key words. stars: abundances - galaxies: dwarf - galaxies: evolution - Local Group - galaxies: formation

\section{Introduction}

Carbon and nitrogen are among the most abundant metals in the Universe and they have a fundamental role in the chemical evolution of galaxies. Carbon is produced in stars of all masses, essentially by helium burning. Nitrogen is synthesised during hydrogen burning through the CNO cycle. While some $\mathrm{N}$ production is predicted in massive stars by mixing between the helium-burning and the hydrogen-burning layers, a large amount of $\mathrm{N}$ is made in intermediate-mass stars of 4 to $11 M_{\odot}$, which undergo hot-bottom burning (Renzini \& Voli 1981; Siess 2010). The abundances of both $\mathrm{C}$ and $\mathrm{N}$ are changed significantly as the stars evolve along the red giant branch (RGB) because of internal mixing, which brings material processed through the $\mathrm{CNO}$ cycle in the stellar interiors to the photosphere. As a result, in evolved RGB stars, $\mathrm{C}$ is depleted and $\mathrm{N}$ is enhanced relative to main- sequence or subgiant branch stars that had the same

\footnotetext{
* Based on observations made with ESO Telescopes at the La Silla Paranal Observatory under programme ID 091.D-0089
}

chemical composition at birth. Therefore, the interpretation of the observations in terms of evolution of both elements needs to account for these stellar evolution effects.

While the abundance properties of $\mathrm{C}$ and $\mathrm{N}$ in individual stars in globular clusters (GCs) have been widely explored, little is known of these properties in galactic systems of not too dissimilar a stellar mass, i.e., the early-type dwarf galaxies that surround the Milky Way (MW). Given the abundance of dwarf galaxies in the Local Group, which is conveniently placed at a relatively close distance to us, using current facilities the stellar populations of these galaxies can be studied on a star-by-star basis. They are also the galactic environments with the lowest mean metallicity. Thus, the study of their stellar populations gives us the possibility to learn about star formation processes in the most pristine environments we can observe (Tolstoy et al. 2009), acting as the local, accessible counterparts of high-redshift objects.

Sculptor is one of the most studied dwarf spheroidal (dSph) galaxies. It is relatively faint $\left(M_{\mathrm{V}} \simeq-11.2\right)$ and located at a distance of $86 \pm 5 \mathrm{kpc}$ (Pietrzyński et al. 2008). Star formation 
in this galaxy took place for an extended period of time, although it mostly contains stars older than 10 Gyr (de Boer et al. 2012). This makes Sculptor an ideal candidate to study the earliest phases of star formation and chemical enrichment processes. As such, a number of large spectroscopic surveys have been carried out in this galaxy. Metallicities $([\mathrm{Fe} / \mathrm{H}])$ from intermediate resolution spectroscopy of several hundred RGB stars, which are probable members of Sculptor, were determined by Tolstoy et al. (2004) and Battaglia et al. (2008) out to its nominal tidal radius. An extended sample of Scl stars is presented in Starkenburg et al. (2010). Kirby et al. (2009) derived abundances for Fe, Mg, Si, $\mathrm{Ca}$, and $\mathrm{Ti}$ from medium-resolution $(R \simeq 6500)$ spectroscopy for 388 radial velocity member stars within $\sim 2$ core radii. Abundances have been measured for a range of elements, including $\mathrm{Fe}, \mathrm{Ca}, \mathrm{Mg}$, Ti, Y, Ba and Eu for about 90 stars in Sculptor from high-resolution FLAMES/GIRAFFE observations in the central regions (Tolstoy et al. 2009; Hill et al. in prep.)

Additionally, carbon has been derived in many giants in this dSph. Azzopardi et al. (1985, 1986) survey Sculptor for carbon- and $\mathrm{CH}$-stars, but they did not quantify their carbon enhancement (see also Groenewegen et al. 2009). Shetrone et al. (1998) measured low-resolution [C/Fe] abundances for two stars in Sculptor, confirming that the CN strong star found by Smith \& Dopita (1983) exhibits both strong CN and CH bands compared to other stars with similar atmospheric parameters, but does not show strong $\mathrm{C}_{2}$ absorptions. They conclude that this star is not similar to the $\mathrm{CN}$ strong stars found in $\mathrm{GCs}$, where $\mathrm{CN}$ strong stars usually show weak $\mathrm{CH}$ absorption. High-resolution spectroscopic follow up of the most metal-poor candidate stars in Sculptor was carried out by Tafelmeyer et al. (2010), Frebel et al. (2010), Kirby \& Cohen (2012), Starkenburg et al. (2013b), and Simon et al. (2015); these studies include C measurements. No carbon-enhanced metal poor (CEMP) stars were identified in those studies. Recently, Skúladóttir et al. (2015) estimated carbon abundances and upper limits from near-infrared $\mathrm{CN}$ bands for about 80 Sculptor giants observed with FLAMES/GIRAFFE. They also discovered the first CEMP-no star in Scl, i.e., with no overabundance of n-capture elements. Finally, Kirby et al. (2015) present $[\mathrm{C} / \mathrm{Fe}]$ abundances for several globular clusters and dSph RGB stars, including 198 stars in Sculptor. They used these abundances to study evolutionary effects on carbon abundances in two different environments (i.e., dSph and GCs), Sculptor chemical enrichment, and its relation with the Galactic halo.

In this work, we report carbon and nitrogen abundances of 94 RGB stars in Sculptor. This samples adds to the literature C-measurements for 85 new stars; to our knowledge, none of the large spectroscopic surveys of Sculptor have so far included measurements of nitrogen abundances, with the exception of one star in Skúladóttir et al. (2015).

We also use our VIMOS data to carry out the first attempt for a search of dissolved globular clusters in the field of a dwarf spheroidal galaxy via the investigation of chemical anomalies. Only a handful of Local Group dwarf galaxies host GCs at present (van den Bergh 2006); it is however conceivable that a larger fraction of Local Group dwarf galaxies may have once hosted GCs, now in the form of disrupted remnants. This is suggested by the presence of cold-kinematic substructures in several of these objects, i.e. groups of stars with a very low velocity dispersion with respect to the overall population of the galaxy (e.g., in Ursa Minor, Sextans and Sculptor dSphs; Kleyna et al. 2003; Battaglia 2007; Battaglia et al. 2011). Interestingly, the metallicity measurements existing for individual stars in Sculptor and Sextans suggest very low average metallicities
$([\mathrm{Fe} / \mathrm{H}]=-2.0,-2.5 \mathrm{dex})$ for these substructures, which would place them among the most metal-poor stellar clusters known.

The presence of disrupted GCs in dwarf galaxies provides insight into the star formation modes at low metallicity/early times. In the Fornax dSph, a comparison between the metallicity distribution function of field stars and that of the surviving GCs already revealed that one-fifth to one-fourth of all stars in the Fornax dSph with $[\mathrm{Fe} / \mathrm{H}]<-2$ dex belong to the four most metal-poor GCs. This also implies that these GCs could not have been four to five times more massive than at present, which poses difficulties for scenarios of self-enrichment and early evolution of GCs postulating much larger masses for their progenitors (Larsen et al. 2012).

While chemical tagging is a promising route for identifying stars that share very similar chemical signatures (e.g., Karlsson et al. 2012 for an application to Sextans), the so-called unambiguous signature that a star formed in a GC-like environment is the presence of characteristic anticorrelations between light elements such as C, N, O, and Na (see, e.g., Smith \& Norris 1984; Carretta et al. 2009; Martell \& Smith 2009; Martell \& Grebel 2010; and Gratton et al. 2012 for a recent review). These kinds of anticorrelations are currently known to be present in GCs with present-day mass as low as $10^{4} M_{\odot}$ (e.g., Dalessandro et al. 2014), of the order of the stellar mass estimated for the substructures in Sextans and Sculptor. Here we focus on the chemodynamical substructure detected by Battaglia (2007) in Sculptor as a group of stars clustering in a narrow velocity range well distinct from Sculptor systemic velocity at projected elliptical radii $0.18 \lesssim R[\mathrm{deg}] \lesssim 0.35$. We revisit this classification by assigning a probability of membership to the substructure according to the line-of-sight velocity distribution (LOSVD) expected for MW, Sculptor field, and Sculptor substructure stars at $0.18 \leq R[\mathrm{deg}] \leq 0.35$. We model the overall LOSVD as a sum of Gaussians along the lines of studies by Battaglia (2007), but add the substructure as am additional term. This yields an estimated central velocity and dispersion of the Sculptor main body and substructure $v_{\text {main }}=109.8 \mathrm{~km} \mathrm{~s}^{-1}, \sigma_{\text {main }}=7.4 \mathrm{~km} \mathrm{~s}^{-1}$, and $v_{\text {sub }}=133.9 \mathrm{~km} \mathrm{~s}^{-1}$ and $\sigma_{\text {sub }}=3.3 \mathrm{~km} \mathrm{~s}^{-1}$, respectively. Seven stars are identified as probable substructure members: they have an average $\mathrm{CaT}[\mathrm{Fe} / \mathrm{H}]=-1.9 \pm 0.1 \mathrm{dex}$, with a scatter of $0.17 \pm 0.1 \mathrm{dex}$, which is very close to the average $[\mathrm{Fe} / \mathrm{H}]$ error of 0.14 dex. We were able to allocate VIMOS slits on six of these substructure stars, and their location on the colormagnetude diagram (CMD), field-of-view, metallicity versus $R$ and line-of-sight (LOS) velocity versus $R$ is plotted in Fig. 1.

The faintness of the substructure stars (down to $V \sim 19.3$ ) makes it very hard to search for $\mathrm{Na}-\mathrm{O}$ anticorrelations because determining the oxygen abundance from, for example the small oxygen lines around 6300 or $7700 \AA$, would require prohibitive exposure times even on 8-m class telescopes. Next, we focus on the search for $\mathrm{C}$ and $\mathrm{N}$ anticorrelations, following Martell \& Grebel (2010) and Martell et al. (2011) for SEGUE spectra of Milky Way halo stars. To disentangle chemical anomalies due to the stars being born in a GC environment from the modification to the $\mathrm{C}$ and $\mathrm{N}$ abundances induced by mixing in luminous giant stars, it is crucial to also establish the trend of $\mathrm{C}$ and $\mathrm{N}$ abundances for the field population. Then, we build a suitable comparison sample among our 94 RGB stars by selecting Sculptor field RGB stars spanning a similar range in metallicity, effective temperature, and luminosity to those in the substructure.

This paper is organised as follows. Section 2 presents the observational material and data reduction. We outline our analysis procedure in Sect. 3. In Sect. 4 we discuss our results on the 

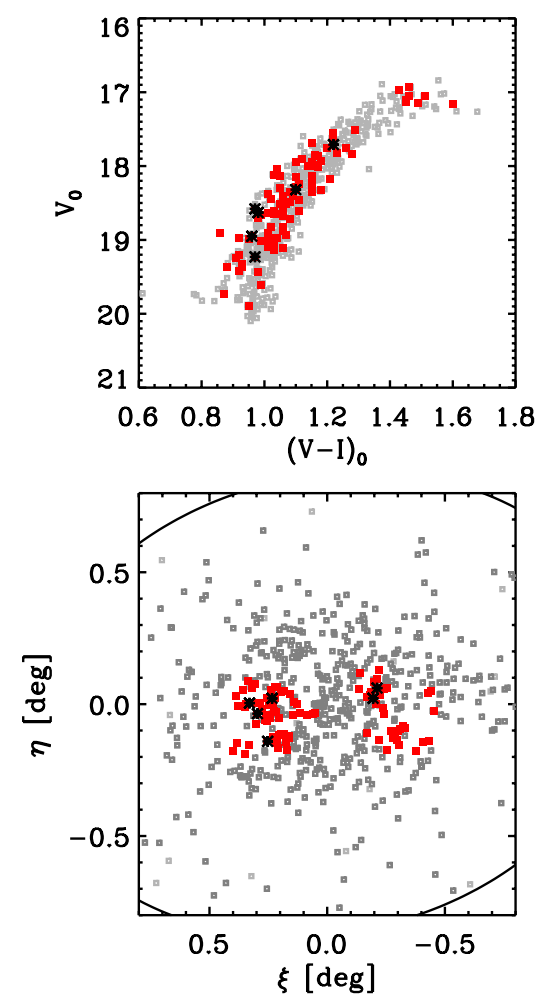
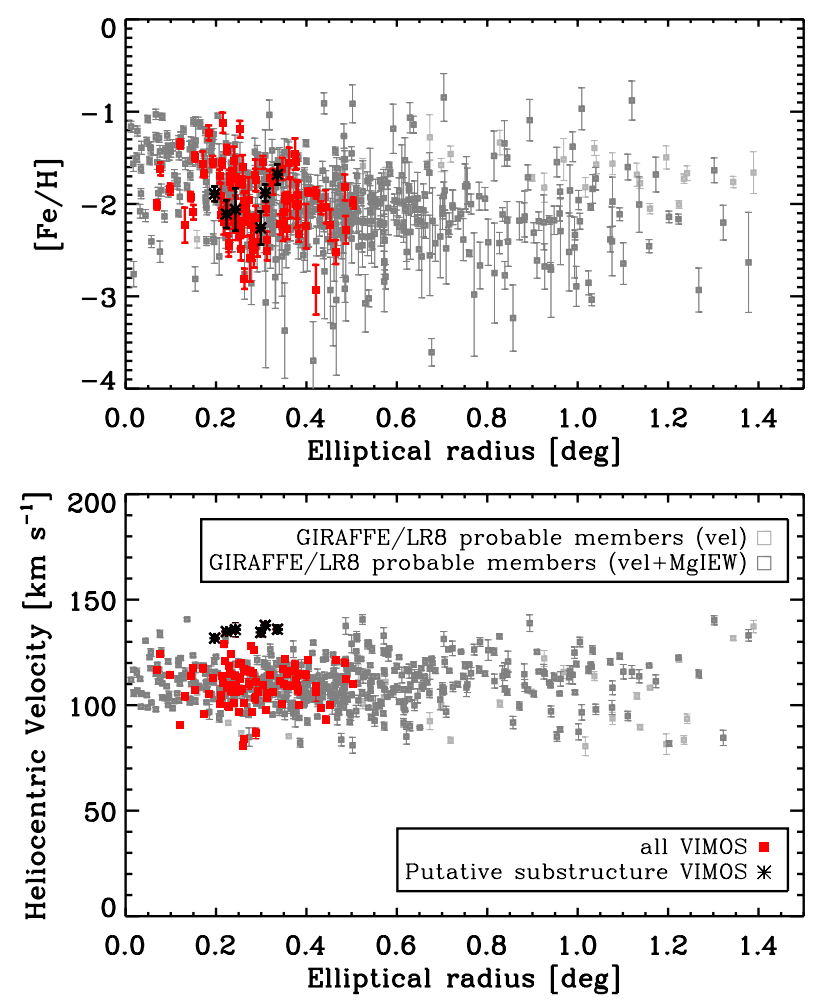

Fig. 1. Properties of our VIMOS sample of highly, likely member stars to Sculptor compared to those of the DART data set of Sculptor stars (see legend). From the top left panel in anticlockwise direction, the location of our VIMOS spectroscopic targets is shown on the Sculptor color-magnitude diagram, field-of-view, LOS velocity versus projected radius and metallicity versus projected radius planes.
C- and N- abundance determinations. We summarise our results and present conclusions in Sect. 5.

\section{Observations and data reduction}

The coordinates, $V$ and $I$ magnitudes, LOS velocities, and $[\mathrm{Fe} / \mathrm{H}]$ of our sample of 94 stars are known from the DART VLT/FLAMES GIRAFFE spectroscopic survey of Sculptor in the region of the NIR Ca II triplet lines (Tolstoy et al. 2004; Battaglia et al. 2008) and from CTIO/MOSAIC II photometric observations (de Boer et al. 2011).

Ninety-two targets are highly likely to be RGB stars members of Sculptor, as indicated by both their LOS velocities and Mg I equivalent width (see Battaglia \& Starkenburg 2012). For the remaining two stars (flagged in Table A.1), the Mg I equivalent width is just outside the range considered by Battaglia \& Starkenburg (2012) for RGB stars; we keep these two objects in the analysis as they do not display properties discrepant from the rest of the sample.

We cannot exclude that our sample contains some contamination from asymptotic giant branch (AGB) stars, as the AGB evolutionary stage merges with the RGB in the CMD. However, it is most likely that all stars in Scl are older than 10 Gyr (de Boer et al. 2012), thus we do not expect severe contamination by intermediate-age AGB stars.

The observations were carried out with VLT/VIMOS in July 2013 in visitor mode, with relatively good atmospheric conditions, e.g., the typical seeing was around 1.0-1.2" . For these MOS observations, we used the HR-blue-(NEW) grism with a slit width of $1.2^{\prime \prime}$, yielding a resolving power $R \simeq 1100$ at $4020 \AA$. The VIMOS field-of-view consists of four quadrants of $7^{\prime} \times 8^{\prime}$ each, separated by a gap of about $2^{\prime}$. For an object at the centre of the quadrant, the wavelength range covered by the HR-blue-(NEW) grism ranges from 3700 to $5240 \AA$.
When planning the observations, we paid particular attention to including the $\mathrm{CN}$-band at $3800 \AA$, in the probed wavelength range of each target.

The location of our VIMOS sample on the Sculptor CMD and field-of-view is shown in Fig. 1, where its metallicity and LOS velocity properties are also compared to the overall population of Sculptor RGB probable members.

We observed four MOS masks for a total integration time of $18.3 \mathrm{~h}$; the signal-to-noise ratio $(\mathrm{S} / \mathrm{N})$ per pixel ranges from 5 to 50 in the $\mathrm{CN}$ feature region (at $3880 \AA$; see Table A.1). Seven stars were observed in more than one mask to test the internal measurement errors. The data reduction was performed with the ESO pipeline ${ }^{1}$ with the standard settings described in the pipeline manual. The pipeline processing steps included the subtraction of the median-combined bias image, creation of the spectral extraction mask from a flat-field image taken immediately following the science exposure, and wavelength calibration constructed from the HeArNe lamp exposures.

We used the radial velocity as derived by the DART VLT/FLAMES GIRAFFE spectroscopic survey (Tolstoy et al. 2004) to correct for radial velocity shifts. We did not attempt to detect possible binary systems by measuring radial velocity from the VIMOS spectra because at this relatively low resolution, the internal error estimated from a cross-correlation against a template, as described in Lardo et al. (2012a), is larger $\left(\simeq 30-40 \mathrm{~km} \mathrm{~s}^{-1}\right)$ than radial velocity variations expected in binary stars. The remaining small shifts are furthermore amended by performing a cross-correlation of the object spectrum with a synthetic spectrum using the most prominent spectral features (e.g., $\mathrm{H} \beta, \mathrm{H} \gamma, \mathrm{H} \delta$, and $\mathrm{Ca} \mathrm{H}+\mathrm{K}$ ) in our wavelength range.

1 http://www.eso.org/sci/software/pipelines/ 

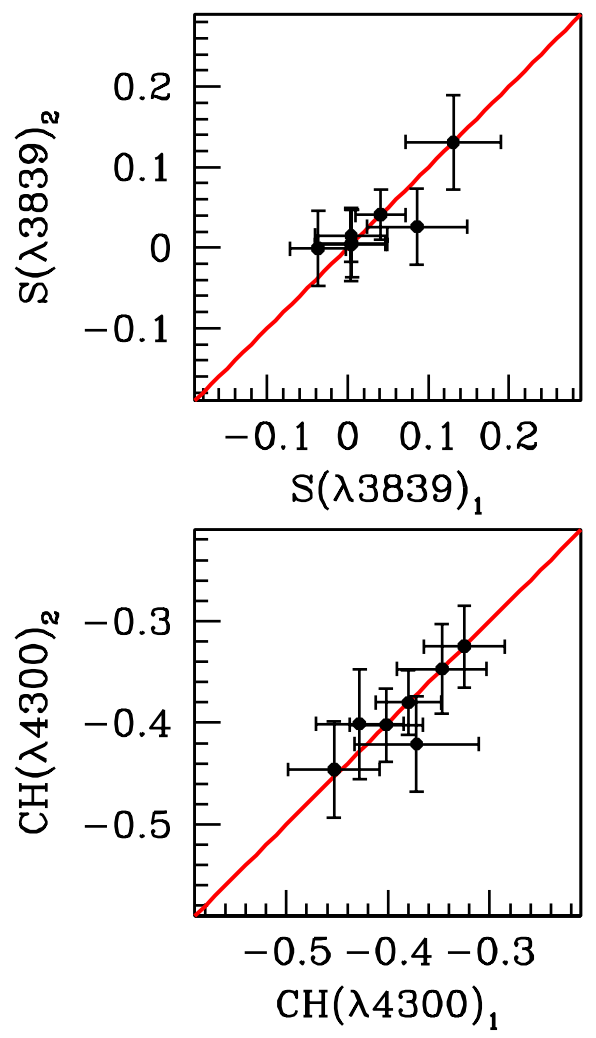

Fig. 2. Comparison of our $\mathrm{CN}$ and $\mathrm{CH}$ measured indices for stars with multiple observations. The red line shows the 1:1 relation.

\section{Indices and abundance analysis}

\subsection{Spectral indices}

In a first approximation, the $\mathrm{CH}(\lambda 4300)$ index can be considered as a carbon-sensitive diagnostic, while the $\mathrm{S}(23839)$ index follows $\mathrm{N}$ abundance. In a star with a solar $\mathrm{C} / \mathrm{N}$ ratio (4.2/1) the nitrogen abundance drives the formation of the $\mathrm{CN}$ band because the molecular abundance is governed by the abundance of the minority species. However, $\mathrm{C}$ and $\mathrm{N}$ abundances are continuously changed by mixing during RGB evolution. As a result, the $\mathrm{C} / \mathrm{N}$ ratio declines while the $\mathrm{CN}$ feature becomes stronger because of the increase of the ${ }^{14} \mathrm{~N}$ abundance. The maximum in the $\mathrm{CN}$ strength is reached when the $\mathrm{C} / \mathrm{N}$ ratio approaches unity. Once the $\mathrm{C} / \mathrm{N}$ ratio decreases below unity, carbon becomes the minority species controlling the $\mathrm{CN}$ band formation so that any additional drop in the $\mathrm{C}$ abundance results in weaker $\mathrm{CN}$ bands. In this section, we measure a set of indices quantifying the strength of the UV/CN band, $\mathrm{S}(\lambda 3839)$ and the $G$ band of $\mathrm{CH}, \mathrm{CH}(\lambda 4300)$.

We adopt the same indices as defined in Pancino et al. (2010) and Lardo et al. (2012a,b, 2013); the uncertainties related to the index measurements are obtained with the expression derived by Vollmann \& Eversberg (2006), assuming pure photon noise statistics in the flux measurements. The indices, together with additional information on the target stars, are listed in Table A.1. As there are seven stars observed in different exposures, we compare the $\mathrm{CH}(\lambda 4300)$ and $\mathrm{S}(\lambda 3839)$ indices obtained from different spectra of the same star. As demonstrated in Fig. 2, the agreement between the index measurements is excellent. This validates both the data reduction and the spectral indices analysis.
The strength of the $\mathrm{S}($ (23839) index strongly depends on the overall metallicity of the star because $\mathrm{CN}$ is a double-metal molecule. Spectroscopic targets in Sculptor span a range of $[\mathrm{Fe} / \mathrm{H}] \simeq 1.8 \mathrm{dex}$ in metallicity. Therefore, we must isolate $\mathrm{C}$ and $\mathrm{N}$ abundance variations from metallicity effects. To this end, we arbitrarily define four metallicity groups: very metal-poor $([\mathrm{Fe} / \mathrm{H}]<-2.2 \mathrm{dex})$, metal-poor $(-2.2 \leq[\mathrm{Fe} / \mathrm{H}]<-1.9 \mathrm{dex})$, intermediate metallicity $(-1.9 \leq[\mathrm{Fe} / \mathrm{H}]<-1.6 \mathrm{dex})$, and metal-rich stars $(-1.6 \leq[\mathrm{Fe} / \mathrm{H}]<-1.0 \mathrm{dex})$. The $\mathrm{S}(\lambda 3839)$ and $\mathrm{CH}(\lambda 4300)$ index measurements in the four metallicity bins are plotted in Fig. 3 against the visual magnitude.

Two stars have prominent $\mathrm{S}(\lambda 3839)$ absorptions in the most metal-poor bin: star 20002 and 90085 with $S(\lambda 3839)=0.50 \pm$ 0.11 and $0.68 \pm 0.14 \mathrm{mag}$, respectively. These stars also have a large $\mathrm{CH}(\lambda 4300)$ index, $\mathrm{CH}(\lambda 4300)=-0.13 \pm 0.23$ and $-0.23 \pm$ $0.12 \mathrm{mag}$, although consistent within $1-\sigma$ with the rest of the Sculptor population in the same metallicity bin. The spectra of the two CN-strong stars are compared to stars of similar stellar atmosphere parameters in Fig. 4. It is clear that the enhanced $\mathrm{CH}$ and $\mathrm{CN}$ index is not an artefact due to, for example, a low $\mathrm{S} / \mathrm{N}$. In the figure, the data quality and some of the spectral features of interest for the present analysis are also apparent. In Sect. 4 we discuss these two objects in more details.

To explore the presence of trends with magnitudes, we perform weighted fits to the data in the index-magnitude plane, when excluding the two outlier stars with high $\mathrm{S}($ (23839) index in the most metal-poor bin (bottom right panel). No statistically significant trend of $\mathrm{CH}(\lambda 4300)$ index with $V$ magnitude is detected (see slopes and errors as given in Fig. 3); while we find a trend of increasing $S(23839)$ index for brighter stars, with the relation being steeper for the highest metallicity bin and flattening at lower metallicity.

The pattern of $\mathrm{C}$ and $\mathrm{N}$ abundances underlying the $\mathrm{CH}$ and $\mathrm{CN}$ band indices of Fig. 3 cannot be interpreted on the basis of band strengths alone because both indices are sensitive to atmospheric parameters and metallicity of the stars. Since we are using the $\mathrm{CN}$ band, the coupling between the $\mathrm{C}$ and $\mathrm{N}$ abundances must also be taken into account. In the following sections, we perform spectral synthesis to disentangle the underlying $\mathrm{C}$ and $\mathrm{N}$ abundances from the $\mathrm{CH}$ and $\mathrm{CN}$ band strengths.

\subsection{Stellar atmosphere parameters}

For the measurement of elemental abundances, we need to know the star's effective temperature $\left(T_{\text {eff }}\right)$, gravity $(\log (g))$, microturbolent velocity $\left(v_{\text {mic }}\right)$, and iron abundance $[\mathrm{Fe} / \mathrm{H}]$. In addition, the determination of the $\mathrm{C}$ and $\mathrm{N}$ abundance is dependent upon the assumed $[\mathrm{O} / \mathrm{Fe}]$ abundance ratio.

In the following, we assume as $[\mathrm{Fe} / \mathrm{H}]$ values and their measurement errors those derived in previous spectroscopic studies of the Sculptor dSph using the semi-empirical calibrations between the equivalent widths of the near-infrared $\mathrm{Ca}$ II triplet lines and $[\mathrm{Fe} / \mathrm{H}]$ tested over the range $-4<[\mathrm{Fe} / \mathrm{H}]<-0.5 \mathrm{dex}$ by Starkenburg et al. (2010).

The photometric $T_{\text {eff }}$ was derived from the Ramírez \& Meléndez (2005b) color-metallicity- $T_{\text {eff }}$ relations for giant stars, adopting the polynomial fit corrections appropriate to the metallicity of each given star. For most stars, we determine $T_{\text {eff }}$ as a weighted average of the temperature derived from the $V-I, V-J$, $V-H, V-K$ relations, $T_{\text {eff,col }}$. The photometry in $V$ and $I$ band comes from deep, wide-area CTIO/MOSAIC II data by de Boer et al. (2011), while the infrared $J H K$ s photometry is from VISTA/VIKING survey data. Of the 94 targets, eight were not present in the de Boer et al. (2011) data since they probably fell 


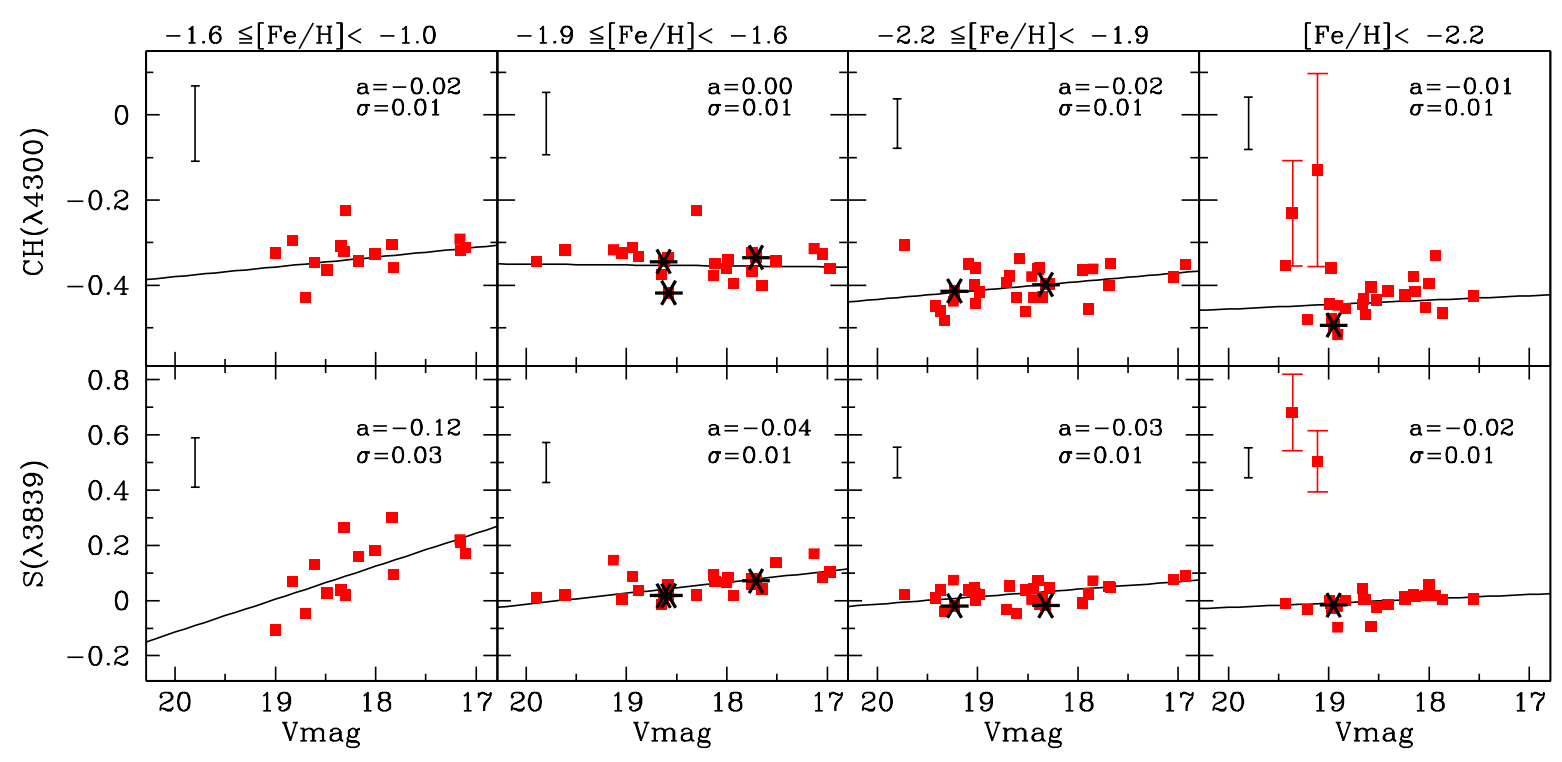

Fig. 3. $\mathrm{CH}(\lambda 4300)$ and $\mathrm{S}(\lambda 3839)$ band-strength index versus extinction-corrected apparent visual magnitude for probable member Sculptor RGB stars in four metallicity bins. Black asterisks denote objects belonging to the kinematic substructure (see text). The range of metallicities considered for each bin is given in the upper part of each panel. We report the mean error associated with index measurements in the top left corner of each panel. Solid lines are best fits to the data when the two notable $\mathrm{S}(\lambda 3839)$ outliers in the most metal-poor bin are excluded. The slope of the best-fit line (a) and its associated error $(\sigma)$ are also reported in the upper right corner of each panel. Error bars for the stars with strong CN absorption are explicitly given in the last panel.

onto bad pixels or were too close to saturated stars; for these, we used previous ESO/WFI $V$ and $I$ photometric data (Tolstoy et al. 2004). The photometry was de-reddened using the Schlegel et al. (1998) maps.

For the $T_{\text {eff }}$ errors in each of the individual color-metallicity$T_{\text {eff }}$ relations, $\sigma_{T_{\text {eff,col }}}$, we sum in quadrature three terms: the first term is derived from the standard error propagation considering the measurement uncertainties in $[\mathrm{Fe} / \mathrm{H}]$, and in the $V$ and $I$ photometry; the second term is the scatter found by Ramírez \& Meléndez (2005b) around the $T_{\mathrm{VI}}-[\mathrm{Fe} / \mathrm{H}]$ color relations; and the third term is the scatter between direct temperature (i.e. stellar angular diameter and bolometric flux measurements) and those derived by the infrared flux method ( $50 \mathrm{~K}$; see Ramírez $\&$ Meléndez 2005a). The final error we quote for $T_{\text {eff }}$ is the error in the weighted average, i.e. $\sigma_{T_{\text {eff }}}=\left(\sum 1 / \sigma_{T_{\text {eff.col }}}^{2}\right)^{-1 / 2}$. For three stars, we could only use the $V-I$ color, as these stars were not present in the available infrared photometric data set. The $\sigma_{T_{\text {eff }}}$ for these stars amounts to $\simeq 60 \mathrm{~K}$, as compared to $\simeq 30 \mathrm{~K}$ for the rest of the sample.

The $\log (g)$ was determined by finding the point along the RGB locus of a set of Dartmouth isochrones ${ }^{2}$ with the closest $T_{\text {eff }},[\mathrm{Fe} / \mathrm{H}],[\alpha / \mathrm{Fe}]$ and age to the values corresponding to a given Sculptor star. We use Dartmouth isochrones of $-2.4 \leq$ $[\mathrm{Fe} / \mathrm{H}] \leq-0.6$ dex and $-0.2 \leq[\alpha / \mathrm{Fe}] \leq+0.6$ dex, both spaced of 0.2 dex. For our sample of Sculptor stars, we assume the trend in $[\alpha / \mathrm{Fe}]$ as a function of $[\mathrm{Fe} / \mathrm{H}]$ observed in a highresolution FLAMES/GIRAFFE pointing (Tolstoy et al. 2009, Hill et al. in prep.) located in the Sculptor central regions, corresponding to $[\alpha / \mathrm{Fe}]=0.33 \mathrm{dex}$ at $[\mathrm{Fe} / \mathrm{H}]<-1.87$ and $[\alpha / \mathrm{Fe}]=$ $-0.5 \times[\mathrm{Fe} / \mathrm{H}]-0.605$ dex at larger $[\mathrm{Fe} / \mathrm{H}]$, and we adopt an error in $[\alpha / \mathrm{Fe}]$ of $\pm 0.1 \mathrm{dex}$. The underlying assumption is that $[\alpha / \mathrm{Fe}]$ as a function of $[\mathrm{Fe} / \mathrm{H}]$ does not vary across the galaxy. This kind of assumption appears to hold for the dSphs for which

\footnotetext{
2 http://stellar.dartmouth.edu/models/index.html
}

this has been verified so far, i.e., Fornax (Letarte et al. 2010; Hendricks et al. 2014; Lemasle et al. 2014). All the Sculptor stars have $[\alpha / \mathrm{Fe}]$ within the isochrone range, while only two are more metal-poor than $[\mathrm{Fe} / \mathrm{H}]=-2.4$ dex outside of their $1 \sigma$ error-bars. de Boer et al. (2012) assigned likely ages to the Sculptor RGB stars with spectroscopic observations, on the basis of the star formation history they derived from deep photometry. This yields ages older than $8 \mathrm{Gyr}$ for the majority of Sculptor stars. We then assigned to the stars in our sample either an age of $8 \mathrm{Gyr}$ or an age of $12 \mathrm{Gyr}$, according to the closest value from de Boer et al. (2012) . The errors in $\log (g)$ were determined by repeating the fit 100 times, where in each of these 100 Montecarlo realisations the $[\mathrm{Fe} / \mathrm{H}], T_{\text {eff }}$ and $[\alpha / \mathrm{Fe}]$ values were randomly extracted from a Gaussian distribution centred on observed values and with standard deviation equal to the measurement error.

For the microturbolent velocity, we used the formula obtained by the GES survey (Gilmore et al. 2012, Bergemann et al., in prep.), which depends on $T_{\text {eff }}, \log (g)$, and metallicity. Here again the errors on $v_{\text {mic }}$ were obtained by standard error propagation.

Table A. 2 reports the $T_{\text {eff }}, \log (g)$ and $v_{\text {mic }}$ values and their uncertainties; these values are used to choose the atmospheric model for the spectral synthesis of each star.

\subsection{Spectral synthesis}

We compared the observed spectra with template synthetic spectra to derive quantitative estimates of $\mathrm{C}$ and $\mathrm{N}$ abundances. The atomic and molecular line lists were taken from the latest Kurucz compilation (Castelli \& Kurucz 2004) and downloaded from F. Castelli's website ${ }^{3}$ both for atomic and molecular transitions. Model atmospheres were calculated with the ATLAS9 code starting from the grid of models available in F. Castelli's

\footnotetext{
3 http://wwwuser.oat.ts.astro.it/castelli/linelists. html
} 


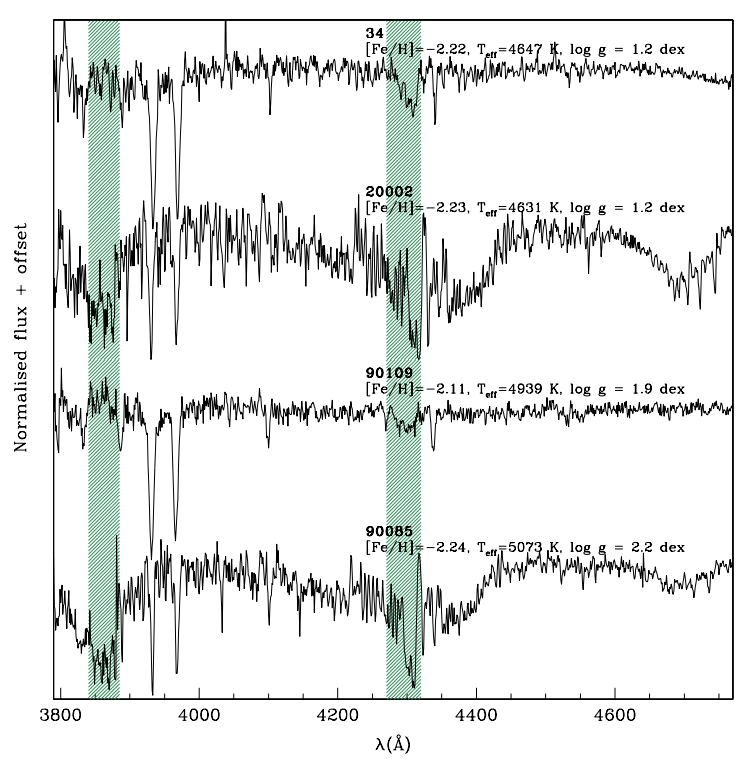

Fig. 4. Comparison between the spectra of the stars with large $\mathrm{C}$ abundance, stars 20002 and 90085, and two sample stars with similar parameters yet very different $\mathrm{CN}$ and $\mathrm{CH}$ absorptions. Also, the narrow $\mathrm{Ca}(\mathrm{H}+\mathrm{K})$ absorption lines indicate very low metallicity $([\mathrm{Fe} / \mathrm{H}] \simeq-2.2 \mathrm{dex})$. The green hatched regions are the windows from which we measured the $\mathrm{CN}$ and $\mathrm{CH}$ indices. The strong features at $4380 \AA$ and $4738 \AA$ are $\mathrm{C}_{2}$ Swan absorptions.

website (Castelli \& Kurucz 2003), using the appropriate values of $[\mathrm{Fe} / \mathrm{H}], T_{\text {eff }}, \log (g)$, and $v_{\text {mic }}$ as derived in the previous section. The ATLAS9 models employed were computed with the new set of opacity distribution functions (Castelli \& Kurucz 2003) and excluded approximate overshooting in calculating the convective flux.

$\mathrm{C}$ and $\mathrm{N}$ abundances were estimated via spectral synthesis of the $\mathrm{CH}$ band at $\simeq 4300 \AA$ and the $\mathrm{CN}$ bands at $\simeq 3883 \AA$, respectively. We computed model spectra by means of the SYNTHE code developed by Kurucz. We derived abundances through a $\chi^{2}$ minimization between the observed spectrum and a grid of synthetic spectra calculated at different abundances. The synthetic spectra have been convolved to the instrumental resolution, then rebinned at the same pixel step as the observed spectra. The upper and middle panels of Fig. 5 illustrate the fit of synthetic spectra to the observed spectra in $\mathrm{CH}$ and $\mathrm{CN}$ spectral regions. Abundances for $\mathrm{C}$ and $\mathrm{N}$ were determined together in an iterative way because for the temperature of our stars, carbon and nitrogen form molecules and, as a consequence, their abundances are related to each other. Reference solar abundances are from Asplund et al. (2009).

To estimate the sensitivity of the derived $A(\mathrm{C})$ and $A(\mathrm{~N})$ abundances $^{4}$ to the adopted atmospheric parameters, we repeated our abundance analysis by changing only one parameter at each iteration (using the errors given in Table A.2) for several stars that are representative of the temperature and gravity range explored. Typically, for the temperature we find $\delta A(\mathrm{C}) / \delta$ $T_{\text {eff }} \simeq 0.05$ dex and $\delta A(\mathrm{~N}) / \delta T_{\text {eff }} \simeq 0.06$ dex. We measure $\delta A(\mathrm{C}) / \delta$ $\log (g) \simeq 0.03$ dex and $\delta A(\mathrm{~N}) / \delta \log (g) \simeq 0.06$ dex for the surface gravity, and $\delta A(\mathrm{C}) / \delta v_{\text {mic }} \simeq 0.02 \mathrm{dex}$ and $\delta A(\mathrm{~N}) / \delta v_{\text {mic }} \simeq$ 0.03 dex for the microturbulent velocity.

The derived abundances are also dependent on the assumed oxygen abundance. Throughout the analysis, we assume the

\footnotetext{
${ }_{4} A(\mathrm{C})$ and $A(\mathrm{~N})$ are the carbon and nitrogen abundances in the usual logarithmic scale, i.e. $A(\mathrm{X})=\log \left(N_{\mathrm{X}} / N_{\mathrm{H}}\right)+12$.
}
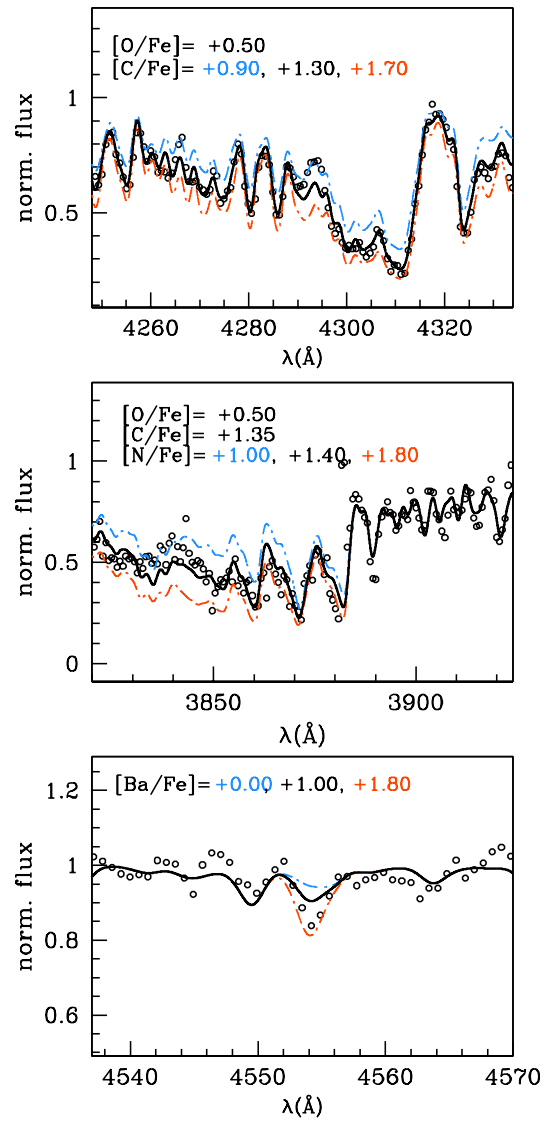

Fig. 5. Observed (black dots) and synthetic (blue, black, and red lines) spectra around the $\mathrm{CH}$ (upper), $\mathrm{CN}$ (middle), and $\mathrm{Ba}$ (bottom) features band for the star $90085(V=19.36, S / N=7$ and 12 at 3880 and $4300 \AA$, respectively) to illustrate the quality of the VIMOS spectra.

$[\mathrm{O} / \mathrm{Fe}]$ versus $[\mathrm{Fe} / \mathrm{H}]$ trend adopted by Skúladóttir et al. (2015) based on the trend observed for a subset of the stars in the highresolution FLAMES/GIRAFFE pointing by Tolstoy et al. (2009) and Hill et al. in prep. We also explored a slightly different trend, where $[\mathrm{O} / \mathrm{Fe}]=[\alpha / \mathrm{Fe}]$; as shown in Fig. 6 this would have had little impact on the final carbon and nitrogen determination, causing a star-to-star random change of about 0.005 dex. As a conservative quantification of the sensitivity of the $\mathrm{C}$ abundance on the adopted $\mathrm{O}$ abundance, we varied the oxygen abundances by as much as 0.4 dex and repeated the spectrum synthesis to determine the exact dependence for the same three representative stars $\left(4100 \mathrm{~K} \leq T_{\text {eff }} \leq 4900 \mathrm{~K}\right)$. We find that changes in the oxygen abundance of 0.4 dex cause a variation in the derived $[\mathrm{C} / \mathrm{Fe}]$ of $\delta \mathrm{A}(\mathrm{C}) /[\mathrm{O} / \mathrm{Fe}] \simeq 0.08 \mathrm{dex}$. This error was then included in the final error computation on $[\mathrm{C} / \mathrm{Fe}]$ and $[\mathrm{N} / \mathrm{Fe}]$.

In addition to the stellar parameters and oxygen abundance errors, a measurement uncertainty exists in the determination of the individual abundances. This intrinsic error was estimated by means of Monte Carlo simulations and combined with the general errors above. Briefly, we repeated the fitting procedure using a sample of 1000 synthetic spectra, where Poissonian noise has been injected (after the re-mapping of the synthetic spectra at the same pixel step of the VIMOS spectra) to reproduce the noise conditions observed around the analysed bands. These uncertainties are of the order of $\simeq 0.09$ and $\simeq 0.23$ dex for $\mathrm{C}$ and $\mathrm{N}$, respectively. All these individual errors were added in quadrature and gave a final error $\Delta[\mathrm{C} / \mathrm{Fe}]=0.14 \mathrm{dex}, \Delta[\mathrm{N} / \mathrm{Fe}]=0.30 \mathrm{dex}$. 

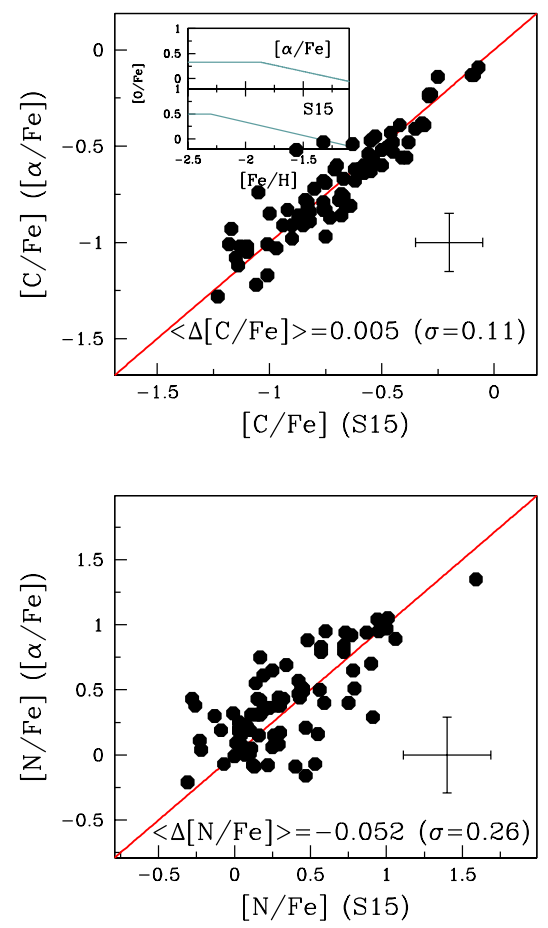

Fig. 6. $[\mathrm{C} / \mathrm{Fe}]$ and $[\mathrm{N} / \mathrm{Fe}]$ abundances computed by setting $[\mathrm{O} / \mathrm{Fe}]=$ $[\alpha / \mathrm{Fe}]$ (denoted with the $[\alpha / \mathrm{Fe}]$ label; see inset) are compared to $[\mathrm{C} / \mathrm{Fe}]$ abundances obtained by assuming the oxygen trend reported in Skúladóttir et al. (2015), denoted with the S15 label. Each panel lists the mean difference with its $\sigma$ in the measured abundances. The red line represents the 1:1 relation.

All the above individual error sources and abundances are listed in Table A.2. We present the abundances derived as described above and the relative uncertainties in Table A.2.

Skúladóttir et al. (2015) estimate [C/Fe] upper limits for 85 Sculptor stars, using VLT/FLAMES intermediate resolution data from the CN molecular line at 9100-9250 A. There are nine stars in our program for which Skúladóttir et al. (2015) report abundances. The latter derive $\mathrm{C}$ estimates and upper limits on the $\mathrm{C}$ abundance from the $\mathrm{CN}$ band, assuming $[\mathrm{C} / \mathrm{N}]=-1.2$ (i.e. the average value for mixed stars in the range $-3 \leq[\mathrm{Fe} / \mathrm{H}] \leq$ -2 dex; Spite et al. 2005) and $[\mathrm{N} / \mathrm{Fe}]=0.0 \mathrm{dex}$; e.g., $[\mathrm{C} / \mathrm{Fe}]_{\text {ext }}$ and $[\mathrm{C} / \mathrm{Fe}]_{\text {lim }}$ of their Table 6 , respectively. We rederived carbon abundances from VIMOS spectra in the CN in the $3880 \AA$ region, under the same assumptions as Skúladóttir et al. (2015), to compare the measured abundances. The comparison is satisfying, with the data scattering about the line corresponding to equality. In most cases, our $\mathrm{C}$ abundances are very close to those by Skúladóttir et al. (2015), mostly within 0.1 dex, with a spread on the whole sample of 0.4 dex. In the case of star 210 , carbon abundances have also been measured from mediumresolution spectra by Kirby et al. (2015). We measure for this star $[\mathrm{C} / \mathrm{Fe}]=-0.75 \pm 0.15 \mathrm{dex}$, which is somewhat higher than the carbon abundances reported by Kirby et al. (2015) and Skúladóttir et al. (2015), $[\mathrm{C} / \mathrm{Fe}]=-0.91$ and -1.01 , respectively, but still within $1-1.5 \sigma^{5}$.

\footnotetext{
5 To compare Kirby et al. (2015) and Skúladóttir et al. (2015) abundances to our data, we shift their abundances to our adopted solar scale (Asplund et al. 2009).
}

\section{Results}

\section{1. $C$ and $N$ abundances and mixing along the $R G B$}

The gathered data set makes it possible for the first time to analyse the $\mathrm{C}$ and $\mathrm{N}$ abundances trends simultaneously in this galaxy over a wide range in $[\mathrm{Fe} / \mathrm{H}]$, and explore the effects of internal mixing along the RGB for stars above the RGB bump.

In Fig. 7 we plot the $[\mathrm{C} / \mathrm{Fe}]$ and $[\mathrm{N} / \mathrm{Fe}]$ abundance ratios for all the observed stars as a function of their $V$ magnitude. We distinguish between four subsamples according to the star's metallicity (see Sect. 3.1). Each panel reports the average $[\mathrm{C} / \mathrm{Fe}]$ and $[\mathrm{N} / \mathrm{Fe}]$ abundances (together with their dispersions), the slope, and the error of the linear fit. Also indicated are the different locations of the RGB bump for the metal-poor and metal-rich populations of Sculptor (Majewski et al. 1999).

Figure 7 shows the decline of $[\mathrm{C} / \mathrm{Fe}]$ with advancing evolution along the RGB (e.g., Shetrone et al. 2013; Kirby et al. 2015). The luminosity-dependent trend detected in Fig. 7 is indicative of some deep mixing, which circulates material from the base of the convective envelope down into the $\mathrm{CN}(\mathrm{O})$-burning region as soon as the star is brighter than the RGB bump (e.g., Charbonnel \& Do Nascimento 1998; Spite et al. 2005). In this sense, the Sculptor stars appear to be behaving in a manner analogous to giants in metal-poor GCs (e.g., Martell et al. 2008b; Shetrone et al. 2010; Kirby et al. 2015), as well as halo field giants of comparable metallicity (Gratton et al. 2000). The carbon depletion appears to be more severe in the bin with the lowest metallicity, i.e. $[\mathrm{Fe} / \mathrm{H}]<-2.2$ dex. This is in agreement with Martell et al. (2008b), who find that the carbon depletion as a function of the time that the star has spent in the deep-mixing phase is approximately twice as large at $[\mathrm{Fe} / \mathrm{H}]=-2.0$ than at $-1.0 \mathrm{dex}$.

In dredged-up material processed by the $\mathrm{CNO}$ cycle, $\mathrm{N}$ is produced at the expense of $\mathrm{C}$. Thus the atmosphere of the star should become $\mathrm{N}$-rich and $\mathrm{C}$-poor relative to its initial composition. On the contrary, the decline in carbon is not accompanied by an increase in $[\mathrm{N} / \mathrm{Fe}]$ abundance with luminosity (lower panels of Fig. 7). The notable exception to this general trend concerns the stars with $[\mathrm{Fe} / \mathrm{H}]<-2.2$ dex, among which there is a trend of increasing $[\mathrm{N} / \mathrm{Fe}]$ with evolving state along the RGB.

To further investigate the behaviour of $\mathrm{C}$ and $\mathrm{N}$ with $\mathrm{RGB}$ evolution, we compare the observed $[\mathrm{C} / \mathrm{Fe}]$ and $[\mathrm{N} / \mathrm{Fe}]$ abundance ratios with the theoretical predictions for evolutionary mixing by Placco et al. (2014) in Fig. 8. The top panels of Fig. 8 show the measured carbon abundances for stars with metallicity $[\mathrm{Fe} / \mathrm{H}]>-2.0$ and $\leq-2.0$ dex (left- and right-hand panel, respectively), along with the Placco et al. (2014) predictions for metallicity $[\mathrm{Fe} / \mathrm{H}]=-1.3$ and -2.3 dex and $[\mathrm{C} / \mathrm{Fe}]=$ $-0.5,+0.0,+0.5,+1.0,+1.5 \mathrm{dex}$, assuming $[\mathrm{N} / \mathrm{Fe}]=+0.0 \mathrm{dex}^{6}$. From this plot, it is apparent that the behaviour of the $[\mathrm{C} / \mathrm{Fe}]$ ratio with the evolutionary stage is perfectly reproduced by the theoretical predictions of Placco et al. (2014) for initial carbon abundance $-0.5 \leq[\mathrm{C} / \mathrm{Fe}] \leq 0.0 \mathrm{dex}$.

The bottom panels of Fig. 8 show the same comparison, but for $[\mathrm{N} / \mathrm{Fe}]$ abundances. The left-hand panel shows models for a metallicity of $[\mathrm{Fe} / \mathrm{H}]=-1.3$ and $[\mathrm{C} / \mathrm{Fe}]=0.0 \mathrm{dex}$ and three different $\mathrm{N}$ abundances. Although the bulk of stars in this wide metallicity bin is significantly metal-poorer than $[\mathrm{Fe} / \mathrm{H}]=-1.3$ dex and is at lower $[\mathrm{C} / \mathrm{Fe}]$ abundances $(\langle[\mathrm{Fe} / \mathrm{H}]\rangle$

6 The Placco et al. models of different $[\mathrm{N} / \mathrm{Fe}]$ predict a very similar behaviour for $[\mathrm{C} / \mathrm{Fe}]$ as a function of $\log (g)$, except for very large $[\mathrm{N} / \mathrm{Fe}]=+2$, values that are absent in our data. 


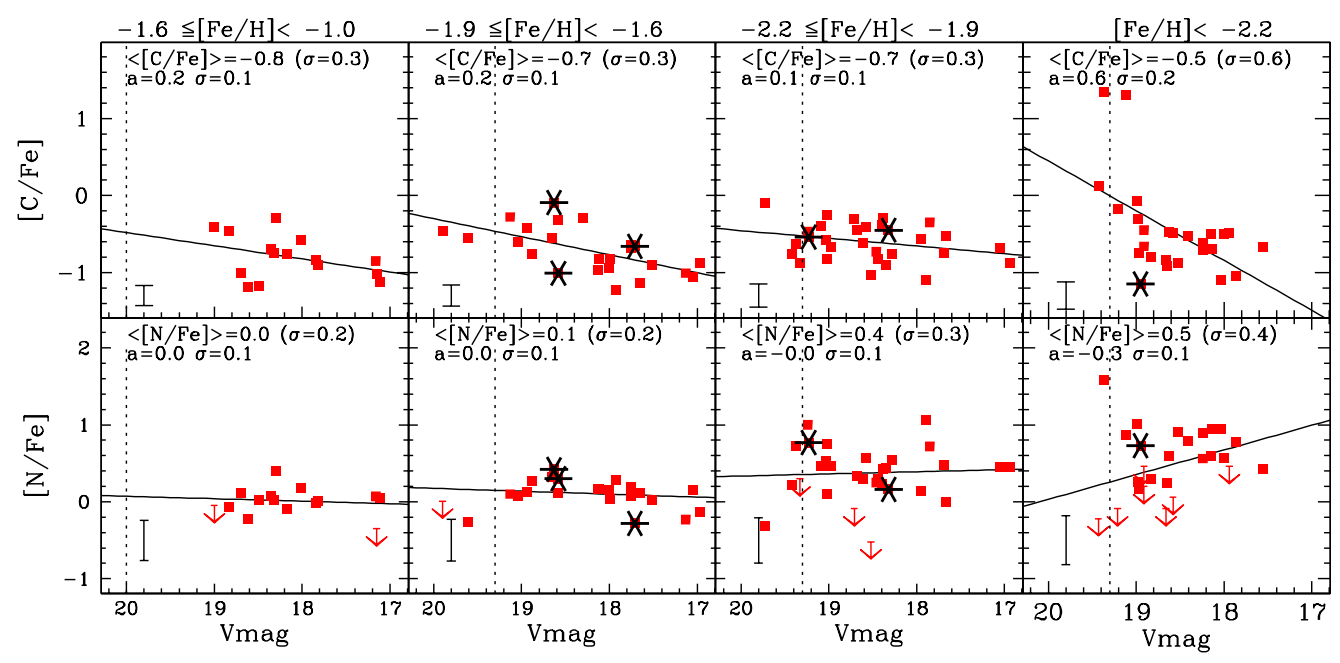

Fig. 7. Run of the $[\mathrm{C} / \mathrm{Fe}]$ and $[\mathrm{N} / \mathrm{Fe}]$ abundance ratio versus extinction-corrected visual magnitude in four bins of metallicity for Sculptor stars, with the black asterisks denoting objects belonging to the kinematic substructure. The vertical line indicates the location of the RGB bump (where deep mixing onsets) for the metal-poor and metal-rich component of Sculptor (see Majewski et al. 1999).
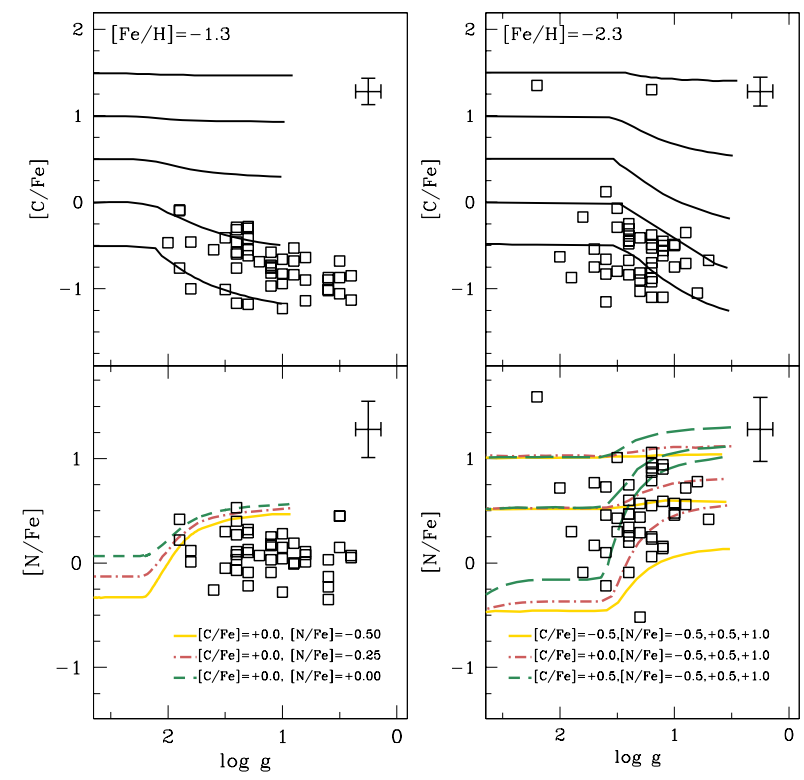

Fig. 8. $[\mathrm{C} / \mathrm{Fe}]($ top) and $[\mathrm{N} / \mathrm{Fe}]$ (bottom) abundance ratios for stars with metallicity $[\mathrm{Fe} / \mathrm{H}]>-2.0$ dex (left-hand panel) and $[\mathrm{Fe} / \mathrm{H}] \leq-2.0$ (right-hand panel) are compared with the theoretical models by Placco et al. (2014) at metallicity $[\mathrm{Fe} / \mathrm{H}]=-1.3$ and $[\mathrm{Fe} / \mathrm{H}]=-2.3$ dex. The mean error on the abundances and the photometric gravity is shown in the top right corner of each panel. Black solid lines (top panel only) are the theoretical models for $[\mathrm{C} / \mathrm{Fe}]=1.5,1.0,0.5,0.0,-0.5$ dex assuming $[\mathrm{N} / \mathrm{Fe}]=+0.0$. Colored lines (bottom panels only) are the theoretical models for $[\mathrm{N} / \mathrm{Fe}]$. The color coding refers to the initial composition of the models; see the keys on the bottom of each plot.

$\simeq-1.7$ and $\langle[\mathrm{C} / \mathrm{Fe}]\rangle \simeq-0.7$ dex, respectively $)^{7}$, theoretical models do not predict a large $[\mathrm{N} / \mathrm{Fe}]$ variation in the surface gravity range explored by VIMOS data. Moreover, the increase of $[\mathrm{N} / \mathrm{Fe}]$ with luminosity, if any, would have been hidden by the large error on $[\mathrm{N} / \mathrm{Fe}]$ abundances. This quantitatively explains

\footnotetext{
7 Qualitatively, we expect that, for metal poor stars, the onset of mixing would be shifted to lower surface gravities and that less initial C leads to smaller $\mathrm{N}$ variations on the upper RGB (see for example Fig. 1 in Placco et al. 2014).
}

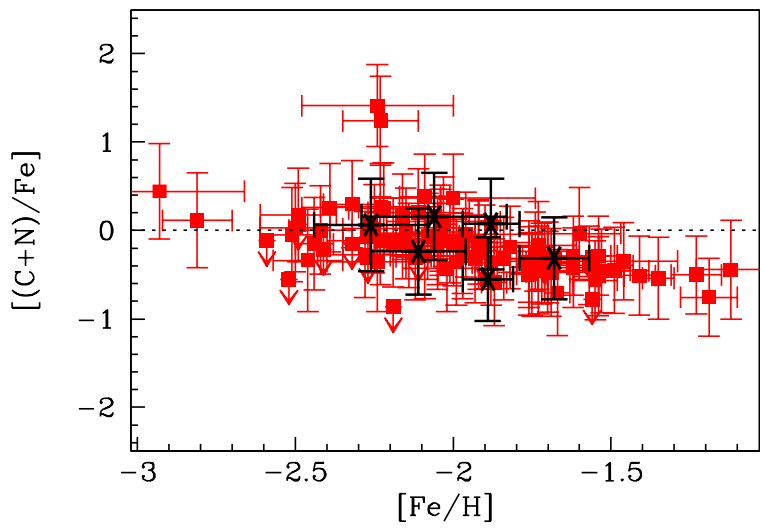

Fig. 9. $[(\mathrm{C}+\mathrm{N}) / \mathrm{Fe}]$ vs. $[\mathrm{Fe} / \mathrm{H}]$ for the spectroscopic sample; symbols are as in Fig. 1. There is a decline of $[(\mathrm{C}+\mathrm{N}) / \mathrm{Fe}]$ with metallicity, an effect previously reported by Spite et al. (2006).

why we do not observe in Fig. 7 a trend of $[\mathrm{N} / \mathrm{Fe}]$ abundances with luminosity for stars more metal-rich than $[\mathrm{Fe} / \mathrm{H}] \simeq-2.0$ dex. In the bottom, right-hand panel, Placco et al. (2014) models for $[\mathrm{Fe} / \mathrm{H}]=-2.3 \mathrm{dex},[\mathrm{C} / \mathrm{Fe}]=-0.5,+0.0,+0.5 \mathrm{dex}$, and initial $\mathrm{N}$ abundance $[\mathrm{N} / \mathrm{Fe}]=-0.5,+0.5,+1.0$ dex are compared with $[\mathrm{N} / \mathrm{Fe}]$ for stars with $[\mathrm{Fe} / \mathrm{H}] \leq-2.0$ dex. From this figure, one can see that the measured $\mathrm{N}$ abundances for stars with $[\mathrm{Fe} / \mathrm{H}] \leq-2.0 \mathrm{dex}$ is well reproduced by theoretical models with $[\mathrm{C} / \mathrm{Fe}]=-0.5,+0.0,+0.5$ dex and metallicity $[\mathrm{Fe} / \mathrm{H}]=$ -2.3 dex. At lower metallicities, Placco et al. (2014) models foresee a larger $\mathrm{N}$-enhancement with advancing evolution. This is consistent with the observed trend of [N/Fe] vs. Vmag observed in Fig. 7 for the most metal-poor stars in our sample.

Finally, Spite et al. (2006) find that the $[(\mathrm{C}+\mathrm{N}) / \mathrm{Fe}]$ ratio is slightly larger for metal-poor stars than for metal-rich stars (see their Fig. 10). This effect is not expected under the assumption that any excess of $\mathrm{N}$ results only from the transformation of $\mathrm{C}$ into $\mathrm{N}$, maybe indicating some metallicity dependence in the efficiency of mixing. Figure 9 shows that a trend of $[(\mathrm{C}+\mathrm{N}) / \mathrm{Fe}]$ with metallicity is also emerging from our data. However, in stars with this luminosity, carbon is highly depleted and the total number of $\mathrm{C}+\mathrm{N}$ atoms is close to the number of $\mathrm{N}$ atoms. 


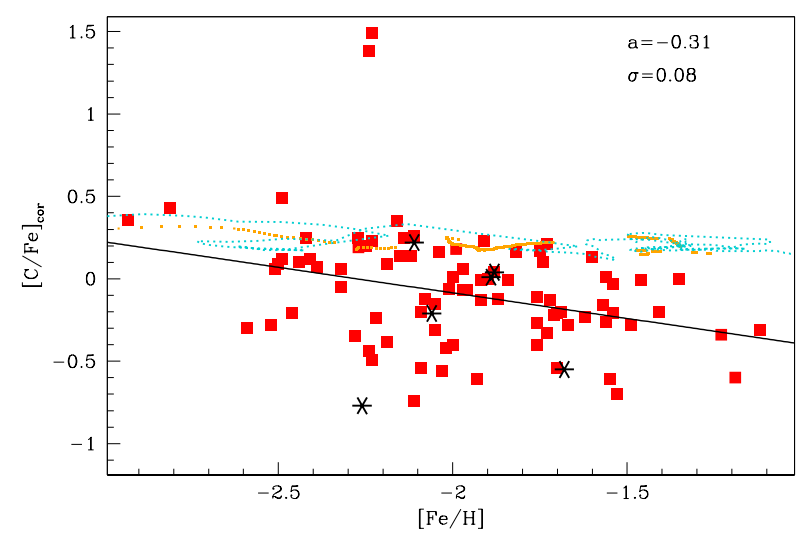

Fig. 10. $[\mathrm{C} / \mathrm{Fe}]$ abundances corrected for luminosity according to Placco et al. (2014) as a function of stellar metallicity. The black solid line is the weighted linear fit to the data. The slope $(a)$ of the fit and the rms $(\sigma)$ are also indicated. The dotted lines are the model presented by Romano \& Starkenburg (2013) (see text).

Therefore, the observed trend of Fig. 9 could also be explained by systematic errors in the $\mathrm{N}$ abundance determinations (NLTE, 3D-effects; e.g., Spite et al. 2005, 2006).

\subsection{Evolution of $C$ and $N$ abundances}

The average values of $[\mathrm{C} / \mathrm{Fe}]$ in the four metallicity ranges of Fig. 7 suggest that the stars with higher metallicity started with lower $[\mathrm{C} / \mathrm{Fe}]$ ratios than stars with $[\mathrm{Fe} / \mathrm{H}] \leq-2.20$ dex (see also Kirby et al. 2015). We recover the initial carbon abundance $\left([\mathrm{C} / \mathrm{Fe}]_{\text {cor }}\right)$ for each star in our sample by applying the corrections computed by Placco et al. (2014), on the basis of models of mixing on the upper RGB that depend on surface gravity, metallicity, and uncorrected carbon abundances. The intent is to explore to what extent the observed trend of $[\mathrm{C} / \mathrm{Fe}]$ with metallicity is due to star-to-star intrinsic variation in carbon abundances rather than to stellar evolution.

Figure 10 confirms that the decline of $[\mathrm{C} / \mathrm{Fe}]$ with increasing $[\mathrm{Fe} / \mathrm{H}]$ in Sculptor cannot be entirely ascribed to stellar evolution. The observed decline in $[\mathrm{C} / \mathrm{Fe}]_{\text {cor }}$ with metallicity can be interpreted by the dependence of the stellar yields on metallicity together with the growing contribution of SN Ia to the interstellar medium enrichment (Kirby et al. 2015). The orange and cyan lines in Fig. 10 show the runs of $[\mathrm{C} / \mathrm{Fe}]$ with metallicity predicted by models $\mathrm{Scl} 2$ and Scl4 of Romano \& Starkenburg (2013), respectively. These authors compute the detailed chemical evolution of Sculptor-like galaxies selected from the mock catalogue of Milky Way satellites by Starkenburg et al. (2013a), by taking into account the IMF-averaged (Salpeter 1955) yields from different nucleosynthetic sources (AGB stars, SNeII and SNeIa), as well as the detailed stellar lifetimes (i.e. the instantaneous recycling approximation is relaxed). More specifically, the adopted stellar yields are from van den Hoek \& Groenewegen (1997) for low- and intermediate-mass stars, Woosley \& Weaver (1995) for massive stars, and Iwamoto et al. (1999) for SNeIa. The star formation in the models is not continuous, but proceeds in bursts. When star formation is not active, we do not plot the corresponding values of the abundance ratios in the interstellar medium, since no stars form with that chemical composition; this is why the model predictions are indicated as broken lines. Figure 10 shows that the upper envelope of the data (when excluding the two C-rich stars) is reasonably well fitted by the models. It is worth emphasising that the goodness of the

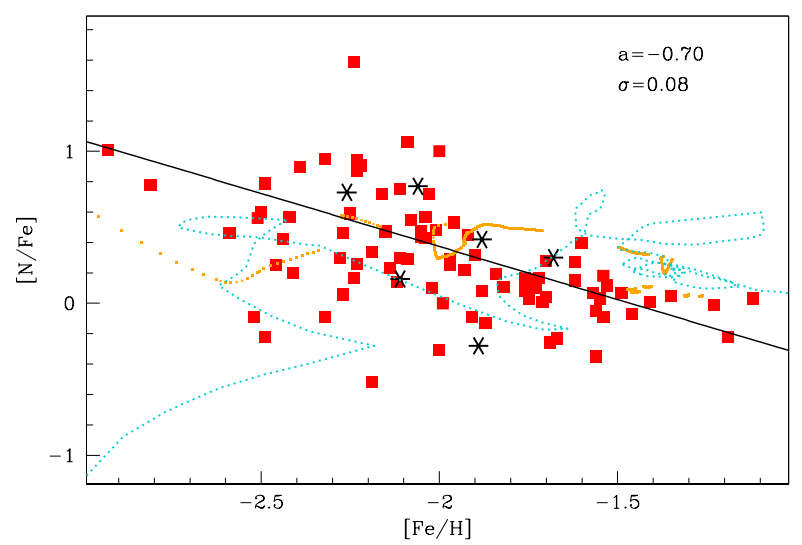

Fig. 11. Same as Fig. 10 but for $[\mathrm{N} / \mathrm{Fe}]$ abundances.

fit obviously depends on the goodness of the adopted yields. Chemical evolution models using the very same set of yields tend to slightly overestimate the $[\mathrm{C} / \mathrm{Fe}]$ ratios of solar neighbourhood stars in the same metallicity range (Romano et al. 2010). Thus, the result for Sculptor is not totally unexpected. It is also worth stressing that the Sculptor models discussed here already nicely reproduce other observed features of this galaxy, such as the $[\mathrm{Mg} / \mathrm{Fe}]-[\mathrm{Fe} / \mathrm{H}]$ relation and its stellar metallicity distribution (see Romano \& Starkenburg 2013)

The $[\mathrm{C} / \mathrm{Fe}]_{\text {cor }}$ abundance ratios of Fig. 10 show a dispersion beyond the measurement uncertainty. When we fit a linear relation between $[\mathrm{C} / \mathrm{Fe}]_{\text {cor }}$ and $[\mathrm{Fe} / \mathrm{H}]$, calculate the residuals about the fit, and divide them by the measurement uncertainties, we find $\Delta=[\mathrm{C} / \mathrm{Fe}]_{\text {cor }} /[\mathrm{C} / \mathrm{Fe}]_{\text {cor }}($ fit $) / \delta[\mathrm{C} / \mathrm{Fe}]=2.1$ (for comparison, Kirby et al. 2015 find 1.5). However, if the observed scatter were due to measurement uncertainties, the value of $\Delta$ would be 1 . Stars in dSph galaxies originate from gas polluted by highenergy supernova ejecta and material from AGB stars of different masses and metallicities, and AGB stars can still pollute the interstellar medium while the galaxy is forming stars. Consequently, the observed residual scatter in carbon abundances can be due to enrichment by various nucleosynthetic sources over time of an interstellar medium, which is not well mixed (Kirby et al. 2015). As pointed out by Kirby et al. (2015), there are several other possible sources for this residual scatter, including inhomogeneous mixing, underestimation of the error on the C abundances, and small errors on the Placco et al. (2014) corrections.

The lower panel of Fig. 7 seems to show that the mean value of $[\mathrm{N} / \mathrm{Fe}]$ decreases with increasing metallicity, perhaps reflecting a decrease in $\mathrm{N}$ production relative to $\mathrm{Fe}$. In principle, the observed $[\mathrm{N} / \mathrm{Fe}]$ decline can be attributed to the low $\mathrm{S} / \mathrm{N}$ ratio of our spectra. However, a plot of $[\mathrm{N} / \mathrm{Fe}]$ abundances vs. $\mathrm{S} / \mathrm{N}$ (not shown) shows that the upper limits on $[\mathrm{N} / \mathrm{Fe}]$ are distributed somewhat evenly in the $[\mathrm{N} / \mathrm{Fe}]-\mathrm{S} / \mathrm{N}$ plane, indicating the presence of a real trend. The $[\mathrm{N} / \mathrm{Fe}]$ values show large scatter and our sample is composed of stars whose surface abundances have been altered by internal mixing. Furthermore, unlike for $\mathrm{C}$-, we cannot access corrections to recover the initial $[\mathrm{N} / \mathrm{Fe}]$, prior to the modifications induced by the mixing. The abundance trends are therefore difficult to verify and interpret. However, from the comparison of the observed $[\mathrm{N} / \mathrm{Fe}]$ ratios with the predictions from models Scl2 and Scl4 by Romano \& Starkenburg (2013) for Sculptor presented in Fig. 11, a general agreement can be found. The model forming stars less efficiently (model Scl2; see Romano \& Starkenburg 2013, their Fig. 2 and 
A\&A 585, A70 (2016)

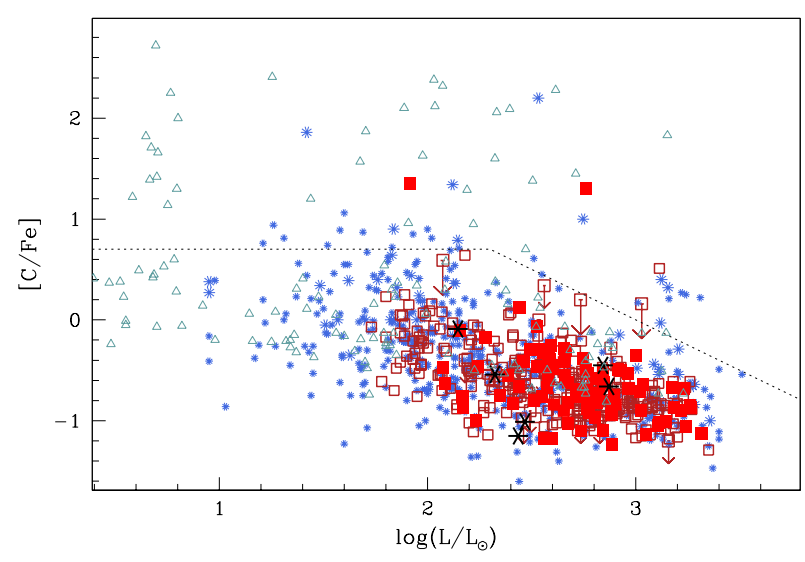

Fig. 12. $[\mathrm{C} / \mathrm{Fe}]$ as a function of $\log \left(L / L_{\odot}\right)$ for Galactic dwarf satellite and halo red giants. The Sculptor stars we analysed are represented by red squares and black asterisks. Burgundy squares denote the $[\mathrm{C} / \mathrm{Fe}]$ abundances of Sculptor stars from the literature (Tafelmeyer et al. 2010; Frebel et al. 2010; Starkenburg et al. 2013b; Skúladóttir et al. 2015; Kirby et al. 2015; Simon et al. 2015). Small light blue asterisks are $[\mathrm{C} / \mathrm{Fe}]$ measurements for $\mathrm{dSph}$ stars from literature data (Fulbright et al. 2004; Cohen \& Huang 2009, 2010; Tafelmeyer et al. 2010; Norris et al. 2010; Lai et al. 2011; Honda et al. 2011; Venn et al. 2012). Empty green triangles represent the halo stars of Yong et al. (2013) with $[\mathrm{Fe} / \mathrm{H}] \geq$ -3.0 dex. The dotted line is the Aoki et al. (2007) dividing line for carbon enhancement, which takes into account the depletion of carbon with evolution along the RGB.

Table 1) does predict a complex behaviour: the [N/Fe] ratio goes up and down, and $[\mathrm{Fe} / \mathrm{H}]$ back and forth, as a consequence of the various episodes of gas accretion onto or loss of gas from the galaxy. The adopted nucleosynthetic yields do not include any primary $\mathrm{N}$ production from massive stars. Thus, according to the models, most of the nitrogen seen in Sculptor would be secondary in nature, coming mostly from intermediate-mass stars that experience hot bottom burning.

\subsection{CEMP stars and the connection with the Galactic halo}

In the MW halo a significant fraction of metal-poor stars, i.e., $[\mathrm{Fe} / \mathrm{H}] \leq-2 \mathrm{dex}$, is enriched in carbon $([\mathrm{C} / \mathrm{Fe}] \geq 0.7 \mathrm{dex})^{8}$. The fraction of CEMP stars also increases with decreasing metallicity (Beers \& Christlieb 2005), suggesting that large amounts of carbon were synthesised in the early Universe when the oldest and most metal-poor stars formed. Traditionally, CEMP stars are divided into two broad categories, according to their chemical composition: carbon enriched stars that display an excess of heavy elements formed in slow $(s)$ or rapid $(r)$ neutron capture processes (CEMP- $s$, CEMP- $r$, and CEMP- $r / s)^{9}$; and CEMP-no, stars that display no such overabundance of neutron-capture elements. The observed chemical pattern of CEMP- $s$ and CEMP$r / s$ stars is thought to be the result of mass transfer in a binary

\footnotetext{
8 Throughout this paper, we adopt the Aoki et al. (2007) criterion, to define carbon-enhanced objects.

9 Barium and europium are often taken as representatives of the $s$ - and $r$-processes. In the solar system, the main component of the $s$-process contributes $\approx 82 \%$ of the $\mathrm{Ba}$, whereas $\approx 18 \%$ is due to $r$ process (Arlandini et al. 1999). On the contrary, the $r$-process accounts for the production of $\approx 94 \%$ Eu. While low mass $\left(M \leq 4 M_{\odot}\right)$ AGB stars are the stellar sources for the $s$-process (Gallino et al. 1998; Arlandini et al. 1999; Travaglio et al. 1999), the $r$-process requires a high-energy, neutron-rich environment, i.e., supernovae (e.g., Travaglio et al. 2004) and/or neutron star mergers (Tsujimoto \& Shigeyama 2014).
}

Table 3. CEMP star frequencies.

\begin{tabular}{clcc}
\hline \hline \multirow{F}{*}{$/ \mathrm{H}] \leq$} & \multicolumn{2}{l}{ \% CEMP stars } & \\
& Scl dSph & Dwarfs & MW Halo \\
\hline-2.0 & $2(3)$ & 8 & 43 \\
-2.5 & $0(4)$ & 22 & 44 \\
-3.0 & $0(8)$ & 40 & 50 \\
-3.5 & $0(17)$ & 50 & 62 \\
\hline
\end{tabular}

Notes. Fraction of CEMP stars (percent; according the Aoki et al. 2007 criterion) per metallicity bins for Scl dSph; dwarf galaxies in the Local Group; and the Galactic halo. The same literature data are plotted in Fig. 12. The values in parenthesis refer to the fraction of CEMP stars in Scl when taking the $[\mathrm{C} / \mathrm{Fe}]$ upper limit from Frebel et al. (2010) into account for star S102549, with $[\mathrm{Fe} / \mathrm{H}]=-3.8$ dex and $[\mathrm{C} / \mathrm{Fe}]=+0.16$ dex.

system, coming from an evolved star in the AGB evolutionary stage (e.g., Lucatello et al. 2005; Starkenburg et al. 2014). In contrast, both CEMP-no and CEMP- $r$ more likely reflect the chemical pattern of the interstellar medium out of which they formed (e.g., Norris et al. 1997; Barklem et al. 2005; Aoki et al. 2007). The significantly distinct binary properties of CEMP-no and CEMP-s stars strongly supports the hypothesis of a different physical origin of their carbon enhancements (Starkenburg et al. 2014).

Carbon measurements have been reported for a significant number of stars in Sculptor, and the available measurements, irrespective of the CEMP star classification, are shown in Fig. 12 together with $[\mathrm{C} / \mathrm{Fe}]$ determinations from literature for $\mathrm{MW}$ dwarf satellites and halo red giants as a function of $\log \left(L / L_{\odot}\right)$. Our $[\mathrm{C} / \mathrm{Fe}]$ determinations nicely fall in the region occupied by the other large samples available in literature (Skúladóttir et al. 2015; Kirby et al. 2015). The great majority of our sample is found to have $[\mathrm{C} / \mathrm{Fe}]<0 \mathrm{dex}$, analogous to that observed by Kirby et al. (2015) for evolved stars in GCs (see for example their Fig. 6). Out of our 94 stars with $[\mathrm{C} / \mathrm{Fe}]$ measurements, we find two stars (2002 and 90085) that are CEMP, i.e., the two $\mathrm{CN}$-strong stars of Sect. 3.1, with around $\simeq 1.0$ dex higher carbon abundance than other stars of similar luminosity or metallicity $\left([\mathrm{C} / \mathrm{Fe}]=1.3\right.$ and $1.35 \mathrm{dex} ;[\mathrm{C} / \mathrm{Fe}]_{\text {cor }}=1.49$ and $1.38 \mathrm{dex}$, respectively). These are also among the most metal-poor stars in the VIMOS sample, with $[\mathrm{Fe} / \mathrm{H}] \simeq-2.2$ dex. Figure 12 also illustrates that so far, in Sculptor, only one other star has been shown to be a CEMP star ${ }^{10}$. This is the CEMP-no star ET0097 $([\mathrm{Ba} / \mathrm{Fe}]<0 \mathrm{dex})$, with metallicity $[\mathrm{Fe} / \mathrm{H}]=-2.03 \pm 0.10$, which is enhanced in carbon and nitrogen by $[\mathrm{C} / \mathrm{Fe}]=0.51 \pm 0.10$ and $[\mathrm{N} / \mathrm{Fe}]=1.18 \pm 0.20$, respectively (Skúladóttir et al. 2015).

To investigate the nature of our two CEMP stars, we estimate their barium abundances, as the strong Ba line at $4554 \AA$ is an accessible indicator of the $s$-processes. Although the low resolution combined with the relatively low $\mathrm{S} / \mathrm{N}$ of our spectra prevent us from measuring $[\mathrm{Ba} / \mathrm{Fe}]$ abundances, both spectra are compatible with having $[\mathrm{Ba} / \mathrm{Fe}]>+1$ dex (see Fig. 5). This finding indicates that both stars might be part of a binary system with a companion star that went through the AGB phase causing pollution with material rich in carbon and $s$-process elements. Then, even though to-date $[\mathrm{C} / \mathrm{Fe}]$ measurements exist for 369 stars, there still remains only one CEMP-no star in Sculptor.

If the stellar halo of our Galaxy was formed long ago out of the shredded stellar component of satellite galaxies resembling the progenitors of MW dSphs, then we would expect the

\footnotetext{
${ }^{10}$ If we exclude the upper limit $[\mathrm{C} / \mathrm{Fe}]$ measurement by Frebel et al. (2010) just above the Aoki et al. (2007) criterion.
} 
oldest stars in both stellar systems to share the same abundance distributions. Currently, there are 118 stars in Sculptor, at metallicity $[\mathrm{Fe} / \mathrm{H}] \leq-2$ dex, with measurements, or upper limits, of [C/Fe] (Frebel et al. 2010; Tafelmeyer et al. 2010; Starkenburg et al. 2013b; Skúladóttir et al. 2015; Kirby et al. 2015; Simon et al. 2015; and this work). In the Galactic halo, the proportion of CEMP stars of all classes is $\approx 40 \%$ (Yong et al. 2013; see Table 3). If this fraction was the same in Sculptor, the expected number of CEMP stars with $[\mathrm{C} / \mathrm{Fe}] \geq 0.7$ dex would be $47 \pm 5$. However, only three CEMP stars have been found, with only the S15 being a CEMP-no star.

As pointed out by Skúladóttir et al. (2015), the comparison with the MW halo becomes much more favourable when focusing on the metallicity range $-2.5 \lesssim[\mathrm{Fe} / \mathrm{H}] \lesssim-2$, where the percentage of CEMP-no halo stars decreases to $5_{-2}^{+3}$. With our sample we add new measurements for other 41 stars in this $[\mathrm{Fe} / \mathrm{H}]$ bin; including the samples from $\mathrm{S} 15$ and $\mathrm{K} 15$, this adds up to 90 stars with measured [C/Fe], of which 3 CEMP stars including only 1 CEMP-no. Therefore in Sculptor, the fraction of CEMP (CEMP-no) stars is $3.4 \%$.

Very recently, Salvadori et al. (2015) used a statistical, datacalibrated cosmological model to investigate the apparent discrepancy between the observed frequency of CEMP stars in the Galactic halo and Sculptor. They find that the fraction of CEMP stars as a function of $[\mathrm{Fe} / \mathrm{H}]$ strongly depends on the luminosity of the dwarf galaxy, but with a fraction approaching unity at $[\mathrm{Fe} / \mathrm{H}] \lesssim-4$ for all objects. For a Sculptor-like galaxy though, whose metallicity distribution function is dominated by stars with $[\mathrm{Fe} / \mathrm{H}] \simeq-2$, the joint probability of observing a star with a given $[\mathrm{Fe} / \mathrm{H}]$ that is also carbon enhanced is maximum in the range $-2.5 \lesssim[\mathrm{Fe} / \mathrm{H}] \lesssim-2$, being $P=0.02$. According to this probability, in a sample of 88 stars we should have observed $1.8 \pm 1.3$ CEMP stars, which is still in agreement with our findings.

\subsection{A disrupted globular cluster?}

In this section we focus on the chemodynamical substructure found by Battaglia (2007) in the Sculptor dSph and use our VLT/VIMOS data to investigate the possibility this may be the remnant of a disrupted globular cluster via searching for the distinctive $\mathrm{C}-\mathrm{N}$ pattern of second-generation globular cluster stars.

Anticorrelations between $[\mathrm{C} / \mathrm{Fe}]$ and $[\mathrm{N} / \mathrm{Fe}]$ are typically detected in GC stars, even in unevolved stars, supposedly not affected by deep mixing (e.g., Cohen et al. 2005). Such anticorrelations can also be detected at low spectral resolution using the $\mathrm{CH}$ and $\mathrm{CN}$ molecular bands as proxies for the $\mathrm{C}$ and $\mathrm{N}$ - abundance, respectively, where stars can be divided in $\mathrm{CN}$-strong/weak, $\mathrm{CH}$-strong/weak groups or show a continuous spread of values (e.g., Pancino et al. 2010). Second-generation GC stars are typically identified with the $\mathrm{CN}$-strong/CH-weak groups. As discussed in the previous sections, abundances of $\mathrm{C}$ and $\mathrm{N}$ in luminous giants are significantly modified by mixing. Furthermore, in our case, we are examining a galactic environment, where the stars have an intrinsic spread in metallicity and age. Since the effects of mixing vary also with metallicity, we would be able to conclude that stars with high $\mathrm{N}$ - and low $\mathrm{C}$ - belonging to the Sculptor substructure are second population GC stars if (at least) a subsample of stars in the substructure will show the strong $\mathrm{CN}$ and enhanced $\mathrm{N}$ abundances relative to the rest of the data set over a similar range of metallicity, color, and magnitude. This line of investigation was successfully adopted by Martell \& Grebel (2010) and Martell et al. (2011) on SEGUE spectra of MW halo stars, finding that $3 \%$ of the observed field MW halo stars show the chemical characteristics of GC stars.

From Figs. 3 and 7, we observe that none of the stars in the kinematic substructure displays either strong $\mathrm{S}(\lambda 3839)$ band strength or large $[\mathrm{N} / \mathrm{Fe}]$ abundances with respect to stars with similar metallicity and magnitude. The $\mathrm{CN}$ band strength is very sensitive to the overall heavy element abundance in the atmosphere, decreasing in abundance roughly proportionally to the decrease in metallicity squared. Thus, in the metal-poor regime, possible metallicity effects may be masking the $\mathrm{C}$ and $\mathrm{N}$ signatures readily found in metal-rich GC stars (e.g., Briley et al. 1993, Smolinski et al. 2011). However, C and N inhomogeneities have been detected in GCs with metallicity comparable to the kinematic substructure in Scl dSph, both in stars fainter and brighter than the RGB bump. In M $15([\mathrm{Fe} / \mathrm{H}]=-2.37 \mathrm{dex})$, stars with luminosities above the RGB bump exhibit in general weak S( 13883 ) CN bands (Trefzger et al. 1983), with a handful of stars having enhanced CN absorption (Langer et al. 1992; Lee 2000). Cohen et al. (2005) find anticorrelated ranges of C and $\mathrm{N}$ abundances in unvolved RGB stars in the same cluster, with large star-to-star differences in $[\mathrm{N} / \mathrm{Fe}]$ abundance ratio up to $\simeq 2.5$ dex. Norris \& Pilachowski (1985) and Smolinski et al. (2011) detect a CN bimodality among stars in $\mathrm{M} 92([\mathrm{Fe} / \mathrm{H}]=$ $-2.31 \mathrm{dex}$ ), although the separation of the populations is minimal, as observed in other metal-poor clusters ( i.e., NGC 5053; see Smolinski et al. 2011). Shetrone et al. (2010) present CN band measurements for 67 stars (both below and up the RGB bump) in NGC $5466([\mathrm{Fe} / \mathrm{H}]=-1.98 \mathrm{dex})$. Their data suggest the presence of a $\mathrm{CN}$ bimodality, although the mean separation in $\mathrm{CN}$ band strength between the two groups is not large relative to the scatter within each group (see also Martell et al. 2008a in the case of $\mathrm{M} \mathrm{55}$, with $[\mathrm{Fe} / \mathrm{H}]=-1.94 \mathrm{dex}$ ).

We tested the hypothesis that the presence of $\mathrm{CN}$-strong, $\mathrm{N}$-rich stars at the low metallicity of the substructure could be hidden by large measurement errors due to the low $\mathrm{S} / \mathrm{N}$ of our spectra in the region of the $\mathrm{CN}$ band. In Figs. 13 and 14 we plot the $S(\lambda 3839)$ band strength and the $[\mathrm{N} / \mathrm{Fe}]$ abundance against the signal-to-noise ratio (per pixel) of two simulated $\mathrm{CN}$-normal and $\mathrm{CN}$-strong stars at a different evolutionary stage (at the RGB bump and at the tip of the RGB). For each $\mathrm{S} / \mathrm{N}$, we simulate $500 \mathrm{CN}$-normal and $500 \mathrm{CN}$-strong spectra with Poissonian noise. For bump stars, we assume $[\mathrm{O} / \mathrm{Fe}]=+0.3$, $[\mathrm{C} / \mathrm{Fe}]=-0.4,[\mathrm{~N} / \mathrm{Fe}]=+0.2 \mathrm{dex}$, and $[\mathrm{O} / \mathrm{Fe}]=+0.4,[\mathrm{C} / \mathrm{Fe}]=$ $-0.8,[\mathrm{~N} / \mathrm{Fe}]=+1.4$ dex for the $\mathrm{CN}$-normal and $\mathrm{CN}$-strong stars, respectively. Those chemical mixtures have been chosen to reproduce the typical ranges in $\mathrm{C}, \mathrm{N}$, and $\mathrm{O}$ abundances observed in unevolved GC stars at low-metallicity (Cohen et al. 2005). As a result of evolutionary effects during the RGB, we expect the carbon abundance to decrease in value with increasing luminosity for stars located on the upper RGB (e.g., Gratton et al. 2000). Therefore for the representative tip star, we adopt $[\mathrm{O} / \mathrm{Fe}]$ $=+0.4 \mathrm{dex},[\mathrm{C} / \mathrm{Fe}]=-1.0 \mathrm{dex},[\mathrm{N} / \mathrm{Fe}]=+0.8 \mathrm{dex}$, and $[\mathrm{O} / \mathrm{Fe}]$ $=+0.4,[\mathrm{C} / \mathrm{Fe}]=-1.4,[\mathrm{~N} / \mathrm{Fe}]=+1.8 \mathrm{dex}$ for the $\mathrm{CN}$-normal and $\mathrm{CN}$-strong stars, respectively. In addition, we explore three metallicity regimes $[\mathrm{Fe} / \mathrm{H}]=-2.5,-2.0$, and -1.5 dex (i.e., the metallicity range covered by our data). Finally, to compute synthetic spectra, we assume $T_{\text {eff }}=5000 \mathrm{~K}, \log (g)=2.0 \mathrm{dex}$, and $v_{\text {mic }}=1 \mathrm{~km} \mathrm{~s}^{-1}$ for the bump star, while for a star near the tip we adopt $T_{\text {eff }}=4000 \mathrm{~K}, \log (g)=0.5 \mathrm{dex}$, and $v_{\text {mic }}=1 \mathrm{~km} \mathrm{~s}^{-1}$.

As expected, from Figs. 13 and 14, we observe that even large variations in the $[\mathrm{N} / \mathrm{Fe}]$ abundance (as large as $\Delta[\mathrm{N} / \mathrm{Fe}] \simeq 1.0-1.2 \mathrm{dex}$ ) reflect a small separation between the $\mathrm{CN}$-normal/N-poor and $\mathrm{CN}$-strong/N-rich stars in the metal poor regime $([\mathrm{Fe} / \mathrm{H}] \leq-2.0 \mathrm{dex})$. However, given the error bars, the 


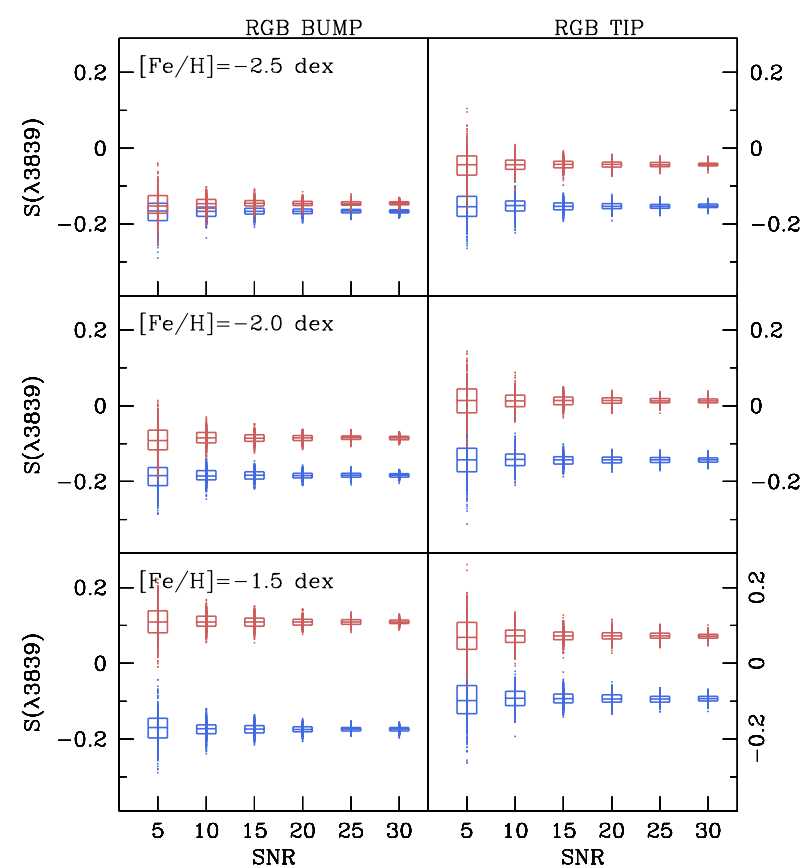

Fig. 13. Box and whisker plot of $\mathrm{S}(\lambda 3839)$ abundances as a function of the signal-to-noise ratio (per pixel) for a $\mathrm{CN}$-normal (blue) and $\mathrm{CN}$ strong (red) simulated stars. Left-hand panels are the results of template synthetic spectra with typical atmospheric parameters as for RGB bump stars, while right-hand panels indicate a representative star at the tip of the RGB. For each evolutionary stage, we report results for three different metallicities, $[\mathrm{Fe} / \mathrm{H}]=-2.5,-2.0$, and -1.5 (from top to bottom). For a given $\mathrm{S} / \mathrm{N}$, the bottom and top of the box represents the $25 \%$ and $75 \%$ percentile range, respectively, and the median is indicated by the band inside the box. The vertical tails extending from the boxes indicate the total range of $\mathrm{S}(23839)$ measurements determined for each $\mathrm{S} / \mathrm{N}$.

separation between the $\mathrm{CN}$-normal and $\mathrm{CN}$-strong stars can already be seen at metallicities $\geq-2.0$ in the indexes of Fig. 13; on the other hand, the larger measurement errors connected to the determination of $\mathrm{N}$ abundances from the spectral synthesis reflect into a less significant separation between the groups of $\mathrm{N}$-poor and $\mathrm{N}$-rich stars. We conclude that the $\mathrm{S} / \mathrm{N}$ of our data would have been sufficient to observe a (marginal) separation in the populations, at least in the $S(\lambda 3839)$ index, at the very low metallicity characteristic of the chemodynamical substructure as well.

In GCs the present-day typical fraction of $\mathrm{CN}$-normal and $\mathrm{CN}$-rich stars is $\sim 50 \%$, ranging from $\sim 30 \%$ to $70 \%$ (e.g., Pancino et al. 2010; see also Bastian \& Lardo 2015 for a discussion about the fraction of enriched-to-normal stars). For a binomial distribution with a $50 \%$ probability of success, there is only a small, although not statistically negligible chance of not detecting any $\mathrm{CN}$-strong stars (probability $\sim 0.016$ ), which can increase (decrease) to $0.12(0.0007)$ for a $70 \%(30 \%)$ probability of success. Given the uncertainties/intrinsic variations in the fraction of $\mathrm{CN}$-strong and $\mathrm{CN}$-weak stars determined in $\mathrm{GCs}$, we cannot exclude that our nondetection of $\mathrm{CN}$-strong stars may be due to small number statistics.

Furthermore, various scenarios that explain chemical anomalies in GCs as the result of self-enrichment postulate that GCs were originally from 10 to 100 times more massive than at present, where the missing mass would all be formed of first generation stars. These large mass losses are in contrast with recent results on the Fornax dSph (Larsen et al. 2012), which set upper limits of four to five times. Even assuming these large values, if

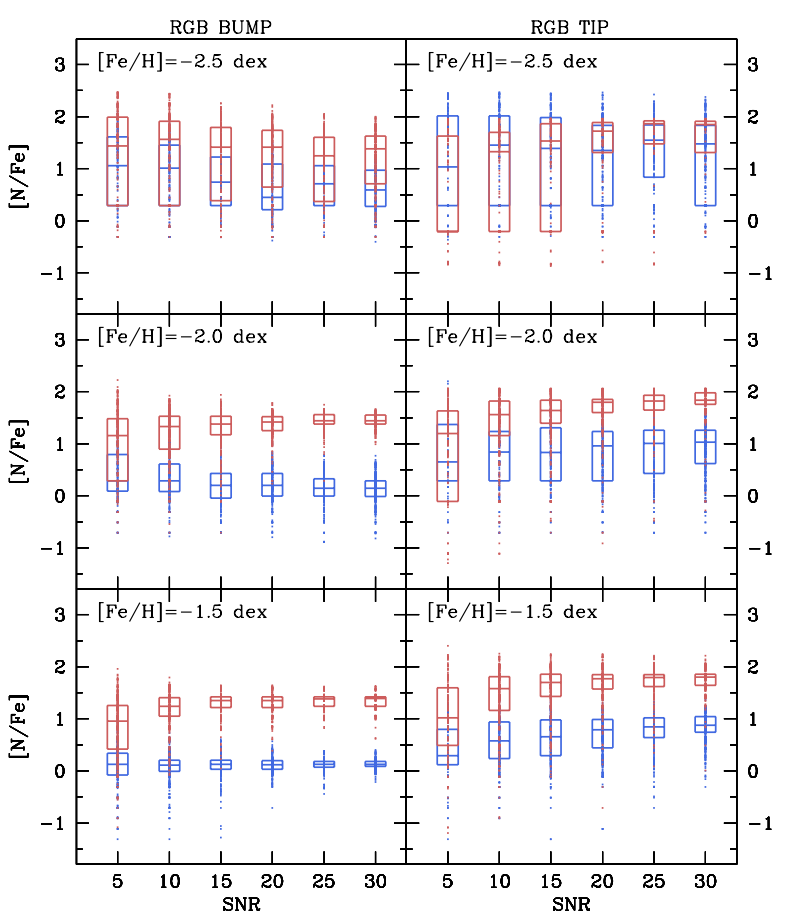

Fig. 14. Same as for Fig. 13, except for [N/Fe] abundances.

the substructure we are seeing is a GC disrupted before it could lose most of the first generation of stars, then the probability of detecting second generation stars would have been even lower. It cannot also be excluded that we are only probing a limited fraction of the stream of tidally shredded GC stars, which could all be made of first generation stars.

Owing to small number statistics and limited spatial coverage as well as uncertainties in our understanding of present-day GC properties and past chemical enrichment, we cannot exclude the possibility that we are observing a disrupted GC.

\section{Summary and conclusions}

We present abundances or upper limits for carbon and nitrogen for 94 RGB stars in Sculptor, derived from low-resolution $(R \simeq 1150)$ spectroscopic observations with VLT/VIMOS. The $\mathrm{C}$ and $\mathrm{N}$ abundances have been derived by fitting model spectra with appropriate atmospheric parameters to the $G$ band at $4300 \AA$ and CN band at $3883 \AA$, respectively. Among these stars, only nine had previously determined carbon abundances. To our knowledge, this is the first time that $\mathrm{N}$ abundances have been measured for a large number of giant stars in a dSph galaxy. The main results of our analysis can be summarized as follows.

We measure the $\mathrm{CH}$ and $\mathrm{CN}$ band strengths and find no significant variations of the $\mathrm{CH}$ index strengths with $V$ magnitude. On the contrary, we notice a trend of increasing $\mathrm{CN}$ index for brighter stars, with the relation being steeper for increasing metallicities.

Carbon shows a decline with increasing luminosity in each metallicity bin. Conversely, nitrogen increases with evolutionary stage along the RGB only in stars more metal-poor than $[\mathrm{Fe} / \mathrm{H}] \leq$ -2.2 dex. We compare the measured abundances with theoretical models to investigate these trends and find excellent agreement between our $[\mathrm{C} / \mathrm{Fe}]$ and $[\mathrm{N} / \mathrm{Fe}]$ abundances and the Placco et al. (2014) theoretical predictions for the range of $\log (g)$ values probed by our target stars. 
The average value of $[\mathrm{C} / \mathrm{Fe}]$, corrected for evolutionary effects, suggests that later forming stars (i.e., more metal-rich stars) started with lower $[\mathrm{C} / \mathrm{Fe}]$ ratios than their predecessors. Additionally, we detect a dispersion in $\mathrm{C}$ abundances that exceeds the scatter expected from measurement uncertainties alone. This can be explained by the pollution from different nucleosynthesis sources over the time of interstellar matter, which additionally has not been well mixed. Although we only qualitatively discuss the evolution of $\mathrm{C}$ abundances, the new data presented here should be used together with literature abundance determinations (Kirby et al. 2015) to make quantitative predictions of the star formation history in Sculptor (following Romano \& Starkenburg 2013).

We identify two new CEMP stars at $[\mathrm{Fe} / \mathrm{H}] \simeq-2.2 \mathrm{dex}$, with original carbon abundance ratios $[\mathrm{C} / \mathrm{Fe}]_{\text {for }}=1.49$ and 1.38 dex. These stars were also identified as $\mathrm{CN}$-strong stars with large $\mathrm{CH}$ absorptions. They are also enriched in $\mathrm{Ba}$ abundance $[\mathrm{Ba} / \mathrm{Fe}]>1.0$ dex, suggesting that both stars have been polluted with a significant amount of carbon and $s$-process elements by an AGB companion. The updated statistics of CEMP and CEMPno stars in Sculptor are still compatible with a framework in which primordial faint supernovae dominated the early chemical enrichment in the MW halo as well as in Sculptor (Salvadori et al. 2015).

Even if further observations are needed to determine elemental abundances of these stars in greater detail, we demonstrated that low-resolution, multiobject spectroscopy of a large number of stars around the UV/CN band can be a quick way to identify candidate CEMP stars for follow-up, high-resolution investigations.

None of the stars belonging to the kinematic substructure uncovered in Scl dSph by Battaglia (2007) displays the chemical C-N signature typical of second populations stars born in GCs. We demonstrated that, even at low metallicity of the substructure, the nondetection of $\mathrm{CN}$-strong, N-rich stars cannot be attributed to insufficient $\mathrm{S} / \mathrm{N}$ ratio if the variations in $[\mathrm{C} / \mathrm{Fe}]$ and $[\mathrm{N} / \mathrm{Fe}]$ in the putative dissolved clusters are as large as those observed in metal-poor Galactic GCs (see Larsen et al. 2014 for $[\mathrm{N} / \mathrm{Fe}]$ variations in the GC system of Fornax). However, because of the uncertainties in the properties and evolution of GCs, as well as to the low number statistics and limited spatial coverage of our sample, we cannot exclude the GC hypothesis. Therefore, additional high-resolution studies designed to measure the key element $\mathrm{Na}$ are needed to establish the nature of this feature.

Acknowledgements. We are grateful to the anonymous referee for his/her careful reading of this manuscript. C.L. and G.B. thank the INAF- Bologna Observatory for their hospitality during part of this work. G.B. gratefully acknowledges support through a Marie-Curie action Intra European Fellowship, funded by the European Union Seventh Framework Program (FP7/2007-2013) under Grant agreement number PIEF-GA-2010-274151, as well as the financial support from the Spanish Ministry of Economy and Competitiveness (MINECO) under the Ramon y Cajal Programme (RYC-2012-11537). D.R. acknowledges financial support from PRIN MIUR 2010-2011, project "The Chemical and Dynamical Evolution of the Milky Way and Local Group Galaxies", prot. 2010LY5N2T. The authors are indebted to the International Space Science Institute (ISSI), Bern, Switzerland, for supporting and funding the international team "First stars in dwarf galaxies".

\section{References}

Aoki, W., Honda, S., Beers, T. C., et al. 2007, ApJ, 660, 747

Arlandini, C., Käppeler, F., Wisshak, K., et al. 1999, ApJ, 525, 886

Asplund, M., Grevesse, N., Sauval, A. J., \& Scott, P. 2009, ARA\&A, 47, 481

Azzopardi, M., Lequeux, J., \& Westerlund, B. E. 1985, A\&A, 144, 388
Azzopardi, M., Lequeux, J., \& Westerlund, B. E. 1986, A\&A, 161, 232 Barklem, P. S., Christlieb, N., Beers, T. C., et al. 2005, A\&A, 439, 129 Bastian, N., \& Lardo, C. 2015, MNRAS, 453, 357

Battaglia, G. 2007, Ph.D. Thesis, Kapteyn Astronomical Institute, University of Groningen

Battaglia, G., \& Starkenburg, E. 2012, A\&A, 539, A123

Battaglia, G., Helmi, A., Tolstoy, E., et al. 2008, ApJ, 681, L13

Battaglia, G., Tolstoy, E., Helmi, A., et al. 2011, MNRAS, 411, 1013

Beers, T. C., \& Christlieb, N. 2005, ARA\&A, 43, 531

Briley, M. M., Smith, G. H., Hesser, J. E., \& Bell, R. A. 1993, AJ, 106, 142

Carretta, E., Bragaglia, A., Gratton, R. G., et al. 2009, A\&A, 505, 117

Castelli, F., \& Kurucz, R. L. 2003, in Modelling of Stellar Atmospheres, eds. N. Piskunov, W. W. Weiss, \& D. F. Gray, IAU Symp., 210, 20

Castelli, F., \& Kurucz, R. L. 2004, ArXiv e-prints [arXiv: astro-ph/0405087]

Charbonnel, C., \& Do Nascimento, Jr., J. D. 1998, A\&A, 336, 915

Cohen, J. G., \& Huang, W. 2009, ApJ, 701, 1053

Cohen, J. G., \& Huang, W. 2010, ApJ, 719, 931

Cohen, J. G., Briley, M. M., \& Stetson, P. B. 2005, AJ, 130, 1177

Dalessandro, E., Massari, D., Bellazzini, M., et al. 2014, ApJ, 791, L4

de Boer, T. J. L., Tolstoy, E., Saha, A., et al. 2011, A\&A, 528, A119

de Boer, T. J. L., Tolstoy, E., Hill, V., et al. 2012, A\&A, 539, A103

Frebel, A., Kirby, E. N., \& Simon, J. D. 2010, Nature, 464, 72

Fulbright, J. P., Rich, R. M., \& Castro, S. 2004, ApJ, 612, 447

Gallino, R., Arlandini, C., Busso, M., et al. 1998, ApJ, 497, 388

Gilmore, G., Randich, S., Asplund, M., et al. 2012, The Messenger, 147, 25

Gratton, R. G., Sneden, C., Carretta, E., \& Bragaglia, A. 2000, A\&A, 354, 169

Gratton, R. G., Carretta, E., \& Bragaglia, A. 2012, A\&ARv, 20, 50

Groenewegen, M. A. T., Lançon, A., \& Marescaux, M. 2009, A\&A, 504, 1031

Hendricks, B., Koch, A., Lanfranchi, G. A., et al. 2014, ApJ, 785, 102

Honda, S., Aoki, W., Arimoto, N., \& Sadakane, K. 2011, PASJ, 63, 523

Iwamoto, K., Brachwitz, F., Nomoto, K., et al. 1999, ApJS, 125, 439

Karlsson, T., Bland-Hawthorn, J., Freeman, K. C., \& Silk, J. 2012, ApJ, 759, 111

Kirby, E. N., \& Cohen, J. G. 2012, AJ, 144, 168

Kirby, E. N., Guhathakurta, P., Bolte, M., Sneden, C., \& Geha, M. C. 2009, ApJ 705,328

Kirby, E. N., Guo, M., Zhang, A. J., et al. 2015, ApJ, 801, 125

Kleyna, J. T., Wilkinson, M. I., Gilmore, G., \& Evans, N. W. 2003, ApJ, 588, L21

Lai, D. K., Lee, Y. S., Bolte, M., et al. 2011, ApJ, 738, 51

Langer, G. E., Suntzeff, N. B., \& Kraft, R. P. 1992, PASP, 104, 523

Lardo, C., Milone, A. P., Marino, A. F., et al. 2012a, A\&A, 541, A141

Lardo, C., Pancino, E., Mucciarelli, A., \& Milone, A. P. 2012b, A\&A, 548, A107

Lardo, C., Pancino, E., Mucciarelli, A., et al. 2013, MNRAS, 433, 1941

Larsen, S. S., Brodie, J. P., \& Strader, J. 2012, A\&A, 546, A53

Larsen, S. S., Brodie, J. P., Grundahl, F., \& Strader, J. 2014, ApJ, 797, 15

Lee, S. G. 2000, J. Korean Astron. Soc., 33, 137

Lemasle, B., de Boer, T. J. L., Hill, V., et al. 2014, A\&A, 572, A88

Letarte, B., Hill, V., Tolstoy, E., et al. 2010, A\&A, 523, A17

Lucatello, S., Tsangarides, S., Beers, T. C., et al. 2005, ApJ, 625, 825

Majewski, S. R., Siegel, M. H., Patterson, R. J., \& Rood, R. T. 1999, ApJ, 520, L33

Martell, S. L., \& Grebel, E. K. 2010, A\&A, 519, A14

Martell, S. L., \& Smith, G. H. 2009, PASP, 121, 577

Martell, S. L., Smith, G. H., \& Briley, M. M. 2008a, PASP, 120, 7

Martell, S. L., Smith, G. H., \& Briley, M. M. 2008b, AJ, 136, 2522

Martell, S. L., Smolinski, J. P., Beers, T. C., \& Grebel, E. K. 2011, A\&A, 534, A136

Norris, J., \& Pilachowski, C. A. 1985, ApJ, 299, 295

Norris, J. E., Ryan, S. G., \& Beers, T. C. 1997, ApJ, 488, 350

Norris, J. E., Wyse, R. F. G., Gilmore, G., et al. 2010, ApJ, 723, 1632

Pancino, E., Rejkuba, M., Zoccali, M., \& Carrera, R. 2010, A\&A, 524, A44

Pietrzyński, G., Gieren, W., Szewczyk, O., et al. 2008, AJ, 135, 1993

Placco, V. M., Frebel, A., Beers, T. C., \& Stancliffe, R. J. 2014, ApJ, 797, 21

Ramírez, I., \& Meléndez, J. 2005a, ApJ, 626, 446

Ramírez, I., \& Meléndez, J. 2005b, ApJ, 626, 465

Renzini, A., \& Voli, M. 1981, A\&A, 94, 175

Romano, D., \& Starkenburg, E. 2013, MNRAS, 434, 471

Romano, D., Karakas, A. I., Tosi, M., \& Matteucci, F. 2010, A\&A, 522, A32

Salpeter, E. E. 1955, ApJ, 121, 161

Salvadori, S., Skuladottir, A., \& Tolstoy, E. 2015, MNRAS, 454, 1320

Schlegel, D. J., Finkbeiner, D. P., \& Davis, M. 1998, ApJ, 500, 525

Shetrone, M. D., Briley, M., \& Brewer, J. P. 1998, A\&A, 335, 919

Shetrone, M., Martell, S. L., Wilkerson, R., et al. 2010, AJ, 140, 1119

Shetrone, M. D., Smith, G. H., Stanford, L. M., Siegel, M. H., \& Bond, H. E 2013, AJ, 145, 123

Siess, L. 2010, A\&A, 512, A10

Simon, J. D., Jacobson, H. R., Frebel, A., et al. 2015, ApJ, 802, 93 
A\&A 585, A70 (2016)

Skúladóttir, Á., Tolstoy, E., Salvadori, S., et al. 2015, A\&A, 574, A129

Smith, G. H., \& Norris, J. 1984, AJ, 89, 263

Smith, G. H., \& Dopita, M. A. 1983, ApJ, 271, 113

Smolinski, J. P., Martell, S. L., Beers, T. C., \& Lee, Y. S. 2011, AJ, 142, 126

Spite, M., Cayrel, R., Plez, B., et al. 2005, A\&A, 430, 655

Spite, M., Cayrel, R., Hill, V., et al. 2006, A\&A, 455, 291

Starkenburg, E., Hill, V., Tolstoy, E., et al. 2010, A\&A, 513, A34

Starkenburg, E., Helmi, A., De Lucia, G., et al. 2013a, MNRAS, 429, 725

Starkenburg, E., Hill, V., Tolstoy, E., et al. 2013b, A\&A, 549, A88

Starkenburg, E., Shetrone, M. D., McConnachie, A. W., \& Venn, K. A. 2014 MNRAS, 441, 1217

Tafelmeyer, M., Jablonka, P., Hill, V., et al. 2010, A\&A, 524, A58

Tolstoy, E., Irwin, M. J., Helmi, A., et al. 2004, ApJ, 617, L119
Tolstoy, E., Hill, V., \& Tosi, M. 2009, ARA\&A, 47, 371

Travaglio, C., Galli, D., Gallino, R., et al. 1999, ApJ, 521, 691

Travaglio, C., Hillebrandt, W., Reinecke, M., \& Thielemann, F.-K. 2004, A\&A, 425,1029

Trefzger, D. V., Langer, G. E., Carbon, D. F., Suntzeff, N. B., \& Kraft, R. P. 1983, ApJ, 266, 144

Tsujimoto, T., \& Shigeyama, T. 2014, ApJ, 795, L18

van den Bergh, S. 2006, AJ, 132, 1571

van den Hoek, L. B., \& Groenewegen, M. A. T. 1997, A\&AS, 123, 305

Venn, K. A., Shetrone, M. D., Irwin, M. J., et al. 2012, ApJ, 751, 102

Vollmann, K., \& Eversberg, T. 2006, Astron. Nachr., 327, 862

Woosley, S. E., \& Weaver, T. A. 1995, ApJS, 101, 181

Yong, D., Norris, J. E., Bessell, M. S., et al. 2013, ApJ, 762, 26 
Appendix A: Additional tables

Table A.1. Sample of probable Sculptor members with properties and index measures.

\begin{tabular}{|c|c|c|c|c|c|c|c|c|c|c|c|c|}
\hline ID & $\begin{array}{c}\text { RA } \\
(\mathrm{J} 2000)\end{array}$ & $\begin{array}{c}\text { Dec } \\
(\mathrm{J} 2000)\end{array}$ & $\begin{array}{r}V \\
\text { (mag) }\end{array}$ & $\begin{array}{r}{[\mathrm{Fe} / \mathrm{H}]} \\
(\mathrm{dex})\end{array}$ & $\begin{array}{r}\mathrm{CN} \\
(\mathrm{mag})\end{array}$ & $\begin{array}{r}\mathrm{eCN} \\
(\mathrm{mag})\end{array}$ & $S / N_{\mathrm{CN}}$ & $\begin{array}{r}\mathrm{CH} \\
\text { (mag) }\end{array}$ & $\begin{array}{r}\mathrm{eCH} \\
(\mathrm{mag})\end{array}$ & $S / N_{\mathrm{CH}}$ & Label & Notes \\
\hline 11 & $00: 58: 58.88$ & $-33: 39: 02.5$ & 18.15 & -2.25 & 0.021 & 0.055 & 14 & -0.38 & 0.055 & 26 & & \\
\hline 12 & $00: 58: 56.16$ & $-33: 38: 42.1$ & 18.68 & -2.19 & 0.052 & 0.074 & 10 & -0.379 & 0.065 & 22 & & \\
\hline 15 & $00: 59: 08.60$ & $-33: 42: 29.4$ & 17.95 & -2.12 & -0.01 & 0.08 & 10 & -0.364 & 0.072 & 20 & & \\
\hline 17 & 00:59:04.05 & $-33: 40: 31.4$ & 18.0 & -2.42 & 0.056 & 0.078 & 17 & -0.3965 & 0.077 & 30 & & \\
\hline 19 & $00: 59: 00.27$ & $-33: 43: 14.4$ & 17.94 & -2.27 & 0.018 & 0.079 & 10 & -0.331 & 0.08 & 18 & & \\
\hline 20 & $00: 59: 06.12$ & $-33: 50: 34.2$ & 18.24 & -2.39 & 0.0040 & 0.03 & 26 & -0.422 & 0.029 & 48 & & \\
\hline 21 & $00: 58: 39.96$ & $-33: 48: 09.8$ & 18.58 & -2.27 & -0.094 & 0.056 & 14 & -0.404 & 0.053 & 26 & & \\
\hline 22 & 00:58:19.97 & $-33: 53: 02.4$ & 18.83 & -2.28 & 0.0020 & 0.04 & 19 & -0.456 & 0.033 & 43 & & \\
\hline 25 & 00:58:05.37 & $-33: 39: 57.2$ & 18.58 & -2.04 & 0.123 & 0.076 & 10 & -0.337 & 0.069 & 21 & & \\
\hline 28 & 01:01:29.21 & $-33: 43: 30.1$ & 17.55 & -2.44 & 0.0050 & 0.031 & 25 & -0.425 & 0.03 & 47 & & \\
\hline 30 & $01: 01: 17.80$ & $-33: 39: 14.8$ & 18.61 & -1.97 & -0.045 & 0.051 & 15 & -0.428 & 0.079 & 18 & & \\
\hline 31 & 01:01:31.76 & $-33: 42: 07.1$ & 18.91 & -2.41 & -0.021 & 0.034 & 22 & -0.448 & 0.041 & 34 & & \\
\hline 32 & $01: 01: 23.15$ & $-33: 41: 05.1$ & 18.38 & -2.04 & -0.014 & 0.061 & 13 & -0.359 & 0.073 & 19 & & \\
\hline 34 & $01: 01: 36.83$ & $-33: 37: 46.6$ & 18.52 & -2.22 & -0.023 & 0.043 & 18 & -0.436 & 0.053 & 26 & & \\
\hline 38 & 01:01:52.59 & $-33: 39: 08.7$ & 18.14 & -2.32 & 0.016 & 0.045 & 17 & -0.416 & 0.054 & 26 & & \\
\hline 40 & 01:01:48.10 & $-33: 42: 30.6$ & 18.97 & -2.24 & -0.028 & 0.048 & 16 & -0.48 & 0.045 & 31 & & \\
\hline 42 & 01:01:49.33 & $-33: 43: 02.9$ & 18.44 & -2.09 & 0.044 & 0.056 & 14 & -0.428 & 0.049 & 29 & & \\
\hline 44 & $01: 01: 57.77$ & $-33: 42: 51.0$ & 18.65 & -1.9 & -0.014 & 0.052 & 15 & -0.376 & 0.053 & 27 & & \\
\hline 46 & 01:02:00.00 & $-33: 51: 13.3$ & 17.75 & -1.88 & 0.079 & 0.076 & 10 & -0.324 & 0.074 & 19 & & \\
\hline 47 & 01:01:23.59 & $-33: 44: 58.1$ & 18.98 & -2.05 & 0.023 & 0.03 & 26 & -0.415 & 0.031 & 45 & & 2 \\
\hline 49 & $01: 01: 25.35$ & $-33: 46: 09.6$ & 19.03 & -1.96 & 0.047 & 0.042 & 19 & -0.399 & 0.042 & 33 & & \\
\hline 53 & 01:01:23.19 & $-33: 50: 51.8$ & 18.35 & -2.05 & 0.012 & 0.065 & 12 & -0.429 & 0.057 & 24 & & \\
\hline 56 & 01:01:14.17 & $-33: 43: 59.1$ & 19.02 & -2.11 & 0.027 & 0.037 & 21 & -0.36 & 0.035 & 41 & & \\
\hline 57 & 01:01:11.54 & $-33: 44: 10.0$ & 18.28 & -2.08 & 0.045 & 0.03 & 25 & -0.397 & 0.023 & 60 & & \\
\hline 60 & 01:01:13.18 & $-33: 38: 34.5$ & 18.41 & -2.49 & -0.015 & 0.055 & 14 & -0.414 & 0.059 & 24 & & \\
\hline 61 & 01:01:10.28 & $-33: 38: 37.9$ & 17.85 & -2.16 & 0.072 & 0.065 & 12 & -0.362 & 0.08 & 18 & & \\
\hline 107 & 01:02:04.17 & $-33: 53: 06.6$ & 18.98 & -2.23 & -0.011 & 0.064 & 12 & -0.36 & 0.049 & 29 & & \\
\hline 141 & $00: 59: 28.42$ & $-33: 35: 23.2$ & 18.71 & -1.91 & -0.03 & 0.088 & 9 & -0.394 & 0.078 & 18 & & \\
\hline 155 & $01: 01: 13.63$ & $-33: 50: 31.3$ & 18.63 & -2.5 & 0.0030 & 0.043 & 18 & -0.468 & 0.054 & 26 & & \\
\hline 156 & 01:01:08.14 & $-33: 49: 32.2$ & 19.21 & -2.32 & -0.032 & 0.095 & 8 & -0.481 & 0.076 & 18 & & \\
\hline 158 & $01: 00: 56.41$ & $-33: 49: 47.1$ & 18.65 & -2.46 & 0.0030 & 0.015 & 51 & -0.433 & 0.063 & 22 & & \\
\hline 182 & $00: 58: 48.66$ & $-33: 50: 16.0$ & 18.46 & -1.97 & 0.0040 & 0.064 & 17 & -0.38 & 0.045 & 45 & & 1 \\
\hline 183 & 00:58:55.98 & $-33: 52: 46.0$ & 19.02 & -2.02 & 0.0010 & 0.043 & 18 & -0.442 & 0.035 & 40 & & \\
\hline 197 & $00: 58: 11.51$ & $-33: 51: 04.0$ & 18.59 & -1.82 & 0.056 & 0.042 & 19 & -0.336 & 0.05 & 29 & & \\
\hline 198 & $00: 58: 04.07$ & $-33: 50: 41.7$ & 17.67 & -1.99 & 0.048 & 0.042 & 18 & -0.349 & 0.044 & 32 & & \\
\hline 206 & 01:00:46.21 & $-33: 42: 33.9$ & 18.04 & -2.23 & 0.018 & 0.052 & 15 & -0.452 & 0.041 & 34 & & \\
\hline 208 & 01:00:33.86 & $-33: 44: 54.4$ & 17.75 & -1.84 & 0.06 & 0.053 & 15 & -0.367 & 0.059 & 24 & & \\
\hline 210 & 01:00:24.63 & $-33: 44: 28.9$ & 17.69 & -2.01 & 0.05 & 0.048 & 16 & -0.401 & 0.057 & 25 & & \\
\hline 220 & 00:59:30.49 & $-33: 39: 04.0$ & 17.9 & -2.09 & 0.024 & 0.048 & 16 & -0.455 & 0.041 & 34 & & \\
\hline 10001 & 00:59:07.84 & $-33: 38: 48.0$ & 18.32 & -2.11 & -0.018 & 0.068 & 11 & -0.399 & 0.059 & 24 & sub & \\
\hline 10002 & $00: 59: 12.71$ & $-33: 41: 09.2$ & 17.71 & -1.89 & 0.071 & 0.065 & 12 & -0.335 & 0.058 & 25 & sub & \\
\hline 10003 & $01: 01: 34.56$ & $-33: 44: 37.9$ & 18.95 & -2.26 & -0.016 & 0.035 & 22 & -0.494 & 0.031 & 45 & sub & \\
\hline 10004 & 01:01:16.50 & $-33: 41: 09.8$ & 19.23 & -2.06 & -0.019 & 0.058 & 21 & -0.414 & 0.069 & 33 & sub & \\
\hline 20005 & 01:01:05.21 & $-33: 39: 40.2$ & 18.52 & -2.19 & 0.037 & 0.045 & 17 & -0.463 & 0.053 & 26 & & \\
\hline 20001 & 01:01:01.44 & $-33: 49: 20.4$ & 18.4 & -2.14 & 0.074 & 0.052 & 15 & -0.361 & 0.057 & 25 & & \\
\hline 20002 & $01: 01: 15.03$ & $-33: 50: 02.6$ & 19.11 & -2.23 & 0.504 & 0.111 & 8 & -0.13 & 0.227 & 7 & & \\
\hline 20003 & 01:01:10.35 & $-33: 51: 15.3$ & 19.43 & -2.49 & -0.011 & 0.049 & 16 & -0.355 & 0.071 & 20 & & \\
\hline 30001 & $01: 01: 44.23$ & $-33: 42: 12.3$ & 18.58 & -1.68 & 0.018 & 0.06 & 13 & -0.418 & 0.059 & 24 & sub & \\
\hline 30002 & 01:01:22.15 & $-33: 50: 55.0$ & 18.63 & -1.88 & 0.018 & 0.081 & 9 & -0.345 & 0.088 & 16 & sub & \\
\hline 50001 & $00: 58: 59.03$ & $-33: 44: 47.4$ & 18.3 & -1.6 & 0.022 & 0.187 & 4 & -0.225 & 0.143 & 10 & & \\
\hline 50002 & 01:01:22.23 & $-33: 46: 21.9$ & 17.87 & -2.81 & 0.0030 & 0.017 & 44 & -0.465 & 0.022 & 64 & & \\
\hline 50004 & 01:00:58.23 & $-33: 52: 51.6$ & 19.09 & -2.15 & 0.039 & 0.048 & 16 & -0.35 & 0.057 & 25 & & \\
\hline 90015 & 00:59:11.34 & $-33: 37: 28.0$ & 17.82 & -1.41 & 0.093 & 0.086 & 9 & -0.358 & 0.084 & 17 & & \\
\hline 90018 & $00: 59: 05.64$ & $-33: 34: 42.0$ & 18.91 & -2.59 & -0.097 & 0.087 & 8 & -0.516 & 0.12 & 12 & & \\
\hline 90026 & $00: 59: 20.23$ & $-33: 40: 53.8$ & 18.35 & -1.57 & 0.039 & 0.068 & 11 & -0.307 & 0.084 & 17 & & \\
\hline 90027 & $00: 59: 16.68$ & $-33: 40: 30.2$ & 17.84 & -1.23 & 0.302 & 0.087 & 9 & -0.304 & 0.097 & 15 & & \\
\hline 90029 & $00: 59: 15.13$ & $-33: 39: 43.8$ & 18.01 & -1.54 & 0.18 & 0.08 & 10 & -0.328 & 0.082 & 17 & & \\
\hline 90030 & 00:59:09.55 & $-33: 39: 06.0$ & 18.32 & -1.12 & 0.266 & 0.113 & 7 & -0.32 & 0.099 & 14 & & \\
\hline 90042 & $00: 58: 36.14$ & $-33: 47: 28.8$ & 19.0 & -1.56 & -0.106 & 0.139 & 5 & -0.324 & 0.114 & 13 & & \\
\hline
\end{tabular}

Notes. We label the stars as "sub" if they were considered as part of the metallicity-substructure. $1=\mathrm{MgIEW}$ just beyond the range for Sculptor giants considered in Battaglia \& Starkenburg (2012); 2 = photometric flag suggests nature as extended object; visual inspection of DSS and 2mass images suggests 147 is a blend, while 90109 looks just as a point-source. 
Table A.1. continued.

\begin{tabular}{|c|c|c|c|c|c|c|c|c|c|c|c|c|}
\hline ID & $\begin{array}{c}\mathrm{RA} \\
(\mathrm{J} 2000)\end{array}$ & $\begin{array}{c}\text { Dec } \\
(\mathrm{J} 2000)\end{array}$ & $\begin{array}{r}V \\
(\mathrm{mag})\end{array}$ & $\begin{array}{r}{[\mathrm{Fe} / \mathrm{H}]} \\
(\mathrm{dex})\end{array}$ & $\begin{array}{r}\mathrm{CN} \\
(\mathrm{mag})\end{array}$ & $\begin{array}{r}\mathrm{eCN} \\
(\mathrm{mag})\end{array}$ & $S / N_{\mathrm{CN}}$ & $\begin{array}{r}\mathrm{CH} \\
(\mathrm{mag})\end{array}$ & $\begin{array}{r}\mathrm{eCH} \\
(\mathrm{mag})\end{array}$ & $S / N_{\mathrm{CH}}$ & Label & Notes \\
\hline 90045 & $00: 58: 33.33$ & $-33: 47: 47.8$ & 18.83 & -1.46 & 0.067 & 0.119 & 7 & -0.295 & 0.114 & 13 & & \\
\hline 90050 & $00: 57: 58.22$ & $-33: 43: 51.5$ & 18.66 & -2.52 & 0.044 & 0.045 & 17 & -0.445 & 0.043 & 32 & & \\
\hline 90057 & 00:58:02.10 & $-33: 39: 29.5$ & 19.37 & -2.03 & 0.039 & 0.066 & 12 & -0.461 & 0.062 & 22 & & \\
\hline 90069 & 01:01:39.57 & $-33: 42: 15.9$ & 18.12 & -1.72 & 0.07 & 0.07 & 11 & -0.349 & 0.066 & 22 & & \\
\hline 90070 & 01:01:41.03 & $-33: 38: 44.1$ & 18.88 & -1.62 & 0.036 & 0.075 & 10 & -0.333 & 0.077 & 19 & & \\
\hline 90074 & 01:01:45.83 & $-33: 37: 10.7$ & 19.24 & -2.0 & 0.074 & 0.074 & 11 & -0.438 & 0.087 & 16 & & \\
\hline 90085 & 01:02:00.20 & $-33: 40: 39.5$ & 19.36 & -2.24 & 0.682 & 0.139 & 7 & -0.231 & 0.124 & 12 & & \\
\hline 90094 & 01:01:50.09 & $-33: 53: 43.1$ & 18.99 & -2.93 & -0.0 & 0.038 & 20 & -0.444 & 0.046 & 30 & & \\
\hline 90096 & $01: 01: 36.27$ & $-33: 47: 01.1$ & 18.24 & -2.51 & 0.017 & 0.032 & 24 & -0.421 & 0.028 & 49 & & \\
\hline 90104 & 01:01:19.26 & $-33: 45: 41.7$ & 17.51 & -1.75 & 0.138 & 0.03 & 27 & -0.343 & 0.032 & 45 & & \\
\hline 90109 & 01:01:18.42 & $-33: 42: 47.0$ & 19.33 & -2.11 & -0.039 & 0.034 & 23 & -0.484 & 0.038 & 36 & & 2 \\
\hline 90112 & 01:01:08.55 & $-33: 45: 35.0$ & 17.15 & -1.56 & 0.211 & 0.045 & 18 & -0.318 & 0.03 & 47 & & \\
\hline 90118 & 01:00:49.37 & $-33: 42: 00.6$ & 17.05 & -1.92 & 0.076 & 0.061 & 13 & -0.382 & 0.073 & 19 & & \\
\hline 90121 & 01:01:12.54 & $-33: 41: 03.9$ & 17.93 & -1.7 & 0.019 & 0.033 & 24 & -0.395 & 0.036 & 39 & & \\
\hline 90122 & 01:01:20.09 & $-33: 41: 45.3$ & 18.61 & -1.19 & 0.131 & 0.083 & 13 & -0.347 & 0.062 & 32 & & \\
\hline 90123 & 01:01:15.08 & $-33: 42: 41.8$ & 17.65 & -1.76 & 0.041 & 0.044 & 25 & -0.402 & 0.051 & 39 & & \\
\hline 90130 & 01:01:02.01 & $-33: 39: 28.8$ & 17.99 & -1.7 & 0.083 & 0.069 & 11 & -0.342 & 0.087 & 16 & & \\
\hline 90131 & 01:00:54.17 & $-33: 40: 14.6$ & 17.13 & -1.67 & 0.17 & 0.094 & 8 & -0.314 & 0.105 & 14 & & \\
\hline 90325 & 00:59:08.56 & $-33: 36: 43.9$ & 18.7 & -1.53 & -0.045 & 0.066 & 11 & -0.43 & 0.072 & 20 & & \\
\hline 90343 & 01:00:59.03 & $-33: 51: 11.5$ & 18.13 & -1.76 & 0.092 & 0.07 & 11 & -0.377 & 0.075 & 19 & & 1 \\
\hline 90344 & 01:00:55.72 & $-33: 50: 11.3$ & 19.13 & -1.73 & 0.146 & 0.082 & 10 & -0.317 & 0.111 & 13 & & \\
\hline 90354 & 01:01:11.51 & $-33: 51: 16.6$ & 19.61 & -1.69 & 0.022 & 0.07 & 11 & -0.317 & 0.083 & 17 & & \\
\hline 90355 & 01:01:05.39 & $-33: 49: 23.9$ & 19.9 & -1.71 & 0.012 & 0.142 & 5 & -0.345 & 0.114 & 13 & & \\
\hline 90361 & 01:01:09.30 & $-33: 52: 34.6$ & 18.49 & -1.55 & 0.026 & 0.056 & 14 & -0.364 & 0.089 & 16 & & \\
\hline 90365 & 01:01:45.00 & $-33: 51: 39.0$ & 19.73 & -2.0 & 0.021 & 0.07 & 11 & -0.306 & 0.076 & 19 & & \\
\hline 90368 & 01:01:12.56 & $-33: 51: 07.8$ & 18.94 & -1.74 & 0.087 & 0.09 & 9 & -0.312 & 0.112 & 13 & & \\
\hline 90448 & $00: 58: 43.53$ & $-33: 50: 30.8$ & 18.17 & -1.54 & 0.161 & 0.061 & 13 & -0.343 & 0.063 & 23 & & \\
\hline 90449 & $00: 58: 46.03$ & $-33: 50: 04.6$ & 19.42 & -1.93 & 0.0090 & 0.055 & 24 & -0.4495 & 0.065 & 30 & & \\
\hline 90452 & $00: 58: 41.31$ & $-33: 51: 49.5$ & 16.97 & -1.87 & 0.103 & 0.057 & 14 & -0.362 & 0.059 & 24 & & \\
\hline 90455 & 00:58:50.91 & $-33: 48: 29.4$ & 19.04 & -1.76 & 0.0050 & 0.06 & 18 & -0.325 & 0.056 & 35 & & \\
\hline 90457 & $00: 58: 42.60$ & $-33: 48: 33.4$ & 16.93 & -1.92 & 0.088 & 0.054 & 14 & -0.351 & 0.065 & 22 & & \\
\hline 90458 & $00: 59: 20.65$ & $-33: 48: 56.6$ & 17.05 & -1.73 & 0.085 & 0.06 & 13 & -0.328 & 0.042 & 34 & & \\
\hline 90549 & $01: 00: 26.29$ & $-33: 44: 45.7$ & 18.0 & -1.62 & 0.065 & 0.06 & 13 & -0.362 & 0.058 & 25 & & \\
\hline 90552 & 01:00:50.87 & $-33: 45: 05.2$ & 17.16 & -1.49 & 0.221 & 0.081 & 10 & -0.292 & 0.101 & 14 & & \\
\hline 90553 & $01: 00: 42.50$ & $-33: 44: 23.5$ & 17.11 & -1.35 & 0.17 & 0.08 & 10 & -0.312 & 0.095 & 15 & & \\
\hline
\end{tabular}


C. Lardo et al.: $\mathrm{C}$ and $\mathrm{N}$ abundances in Sculptor dSph

Table A.2. Atmospheric parameters and $\mathrm{C}$ and $\mathrm{N}$ abundances for the sample stars.

\begin{tabular}{|c|c|c|c|c|c|c|c|c|c|c|c|c|c|}
\hline ID & $\begin{array}{r}T \\
(\mathrm{~K})\end{array}$ & $\begin{array}{l}e T \\
(\mathrm{~K})\end{array}$ & $\begin{array}{r}\log (g)-l \\
(\text { dex })\end{array}$ & $\begin{array}{r}\log (g) \\
(\operatorname{dex})\end{array}$ & $\begin{array}{r}\log (g)-u \\
(\operatorname{dex})\end{array}$ & $\left(\mathrm{km} \mathrm{s}^{-1}\right)$ & $\begin{array}{r}e v_{\mathrm{t}} \\
\left(\mathrm{km} \mathrm{s}^{-1}\right)\end{array}$ & $\begin{array}{r}{[\mathrm{O} / \mathrm{Fe}]} \\
(\mathrm{dex})\end{array}$ & $\begin{array}{r}{[\mathrm{C} / \mathrm{Fe}]} \\
(\mathrm{dex})\end{array}$ & $\begin{array}{r}{[\mathrm{C} / \mathrm{Fe}]_{\text {cor }}} \\
(\mathrm{dex})\end{array}$ & $\begin{array}{r}\mathrm{e}[\mathrm{C} / \mathrm{Fe}] \\
(\mathrm{dex})\end{array}$ & $\begin{array}{r}{[\mathrm{N} / \mathrm{Fe}]} \\
(\mathrm{dex})\end{array}$ & $\begin{array}{r}\mathrm{e}[\mathrm{N} / \mathrm{Fe}] \\
(\mathrm{dex})\end{array}$ \\
\hline 11 & 4589 & 31 & 1.0 & 1.1 & 1.2 & 1.07 & 0.02 & 0.5 & -0.5 & 0.2 & 0.16 & 0.59 & 0.31 \\
\hline 12 & 4680 & 31 & 1.2 & 1.4 & 1.4 & 1.09 & 0.01 & 0.48 & -0.45 & 0.09 & 0.16 & 0.34 & 0.31 \\
\hline 15 & 4589 & 31 & 1.0 & 1.1 & 1.2 & 1.07 & 0.01 & 0.44 & -0.56 & 0.14 & 0.15 & 0.14 & 0.29 \\
\hline 17 & 4554 & 31 & 0.9 & 1.0 & 1.0 & 1.05 & 0.01 & 0.5 & -0.5 & 0.25 & 0.17 & 0.57 & 0.3 \\
\hline 19 & 4512 & 31 & 0.8 & 1.0 & 1.0 & 1.06 & 0.02 & 0.5 & -0.49 & 0.25 & 0.16 & 0.46 & u.l. \\
\hline 20 & 4593 & 31 & 1.0 & 1.1 & 1.1 & 1.06 & 0.01 & 0.5 & -0.62 & 0.07 & 0.17 & 0.9 & 0.3 \\
\hline 21 & 4635 & 31 & 1.1 & 1.2 & 1.3 & 1.08 & 0.01 & 0.5 & -0.49 & 0.19 & 0.16 & 0.06 & u.1. \\
\hline 22 & 4770 & 32 & 1.4 & 1.5 & 1.5 & 1.1 & 0.01 & 0.5 & -0.8 & -0.35 & 0.17 & 0.3 & 0.28 \\
\hline 25 & 4635 & 31 & 1.2 & 1.3 & 1.4 & 1.09 & 0.01 & 0.4 & -0.41 & 0.16 & 0.15 & 0.57 & 0.29 \\
\hline 28 & 4409 & 31 & 0.7 & 0.7 & 0.7 & 1.03 & 0.01 & 0.5 & -0.67 & 0.1 & 0.17 & 0.42 & 0.34 \\
\hline 30 & 4679 & 31 & 1.3 & 1.3 & 1.5 & 1.09 & 0.01 & 0.36 & -0.62 & 0.06 & 0.15 & 0.29 & 0.28 \\
\hline 31 & 4740 & 32 & 1.3 & 1.4 & 1.5 & 1.08 & 0.01 & 0.5 & -0.45 & 0.12 & 0.17 & 0.2 & u.l. \\
\hline 32 & 4726 & 31 & 1.4 & 1.5 & 1.6 & 1.1 & 0.01 & 0.4 & -0.29 & 0.16 & 0.15 & 0.43 & 0.29 \\
\hline 34 & 4647 & 31 & 1.1 & 1.2 & 1.3 & 1.08 & 0.01 & 0.5 & -0.88 & -0.24 & 0.16 & 0.91 & 0.33 \\
\hline 38 & 4648 & 31 & 1.1 & 1.2 & 1.3 & 1.07 & 0.01 & 0.5 & -0.7 & -0.05 & 0.17 & 0.95 & 0.29 \\
\hline 40 & 4878 & 32 & 1.6 & 1.7 & 1.8 & 1.12 & 0.01 & 0.5 & -0.75 & -0.44 & 0.25 & 0.17 & 0.35 \\
\hline 42 & 4673 & 31 & 1.2 & 1.3 & 1.4 & 1.09 & 0.01 & 0.43 & -0.82 & -0.2 & 0.15 & 0.29 & 0.29 \\
\hline 44 & 4631 & 31 & 1.2 & 1.3 & 1.4 & 1.08 & 0.01 & 0.32 & -0.55 & 0.0 & 0.14 & 0.32 & 0.27 \\
\hline 46 & 4311 & 31 & 0.6 & 0.8 & 0.8 & 1.1 & 0.02 & 0.31 & -0.64 & 0.04 & 0.14 & 0.08 & 0.28 \\
\hline 47 & 4673 & 66 & 1.2 & 1.4 & 1.5 & 1.09 & 0.02 & 0.4 & -0.67 & -0.15 & 0.15 & 0.47 & 0.31 \\
\hline 49 & 4681 & 32 & 1.3 & 1.4 & 1.6 & 1.1 & 0.02 & 0.35 & -0.58 & -0.07 & 0.15 & 0.53 & 0.28 \\
\hline 53 & 4621 & 31 & 1.1 & 1.3 & 1.3 & 1.08 & 0.01 & 0.4 & -0.9 & -0.31 & 0.15 & 0.44 & 0.29 \\
\hline 56 & 4727 & 32 & 1.3 & 1.4 & 1.6 & 1.09 & 0.01 & 0.44 & -0.25 & 0.26 & 0.16 & 0.75 & 0.3 \\
\hline 57 & 4558 & 65 & 1.0 & 1.2 & 1.3 & 1.08 & 0.03 & 0.42 & -0.76 & -0.12 & 0.15 & 0.55 & 0.29 \\
\hline 60 & 4668 & 31 & 1.1 & 1.2 & 1.3 & 1.07 & 0.01 & 0.5 & -0.53 & 0.12 & .18 & 0.79 & 0.34 \\
\hline 61 & 4481 & 31 & 0.8 & 0.9 & 1.0 & 1.06 & 0.02 & 0.47 & -0.35 & 0.35 & 0.15 & 0.72 & 0.28 \\
\hline 107 & 4696 & 32 & 1.2 & 1.4 & 1.4 & 1.09 & 0.01 & 0.5 & -0.31 & 0.23 & 0.16 & 0.26 & 0.3 \\
\hline 141 & 4638 & 31 & 1.2 & 1.3 & 1.4 & 1.09 & 0.01 & 0.32 & -0.3 & 0.23 & 0.14 & -0.09 & u.l. \\
\hline 155 & 4751 & 32 & 1.3 & 1.4 & 1.5 & 1.08 & 0.01 & 0.5 & -0.48 & 0.09 & 0.18 & 0.6 & 0.3 \\
\hline 156 & 4956 & 33 & 1.8 & 1.8 & 1.9 & 1.13 & 0.01 & 0.5 & -0.17 & 0.06 & 0.18 & -0.09 & u.l. \\
\hline 158 & 4675 & 65 & 1.1 & 1.2 & 1.4 & 1.07 & 0.02 & 0.5 & -0.92 & -0.21 & 0.21 & 0.25 & 0.4 \\
\hline 182 & 4542 & 31 & 1.0 & 1.1 & 1.3 & 1.08 & 0.02 & 0.36 & -0.73 & -0.07 & 0.14 & 0.25 & 0.29 \\
\hline 183 & 4766 & 32 & 1.4 & 1.6 & 1.6 & 1.11 & 0.01 & 0.39 & -0.83 & -0.42 & 0.15 & 0.1 & 0.3 \\
\hline 197 & 4620 & 31 & 1.2 & 1.4 & 1.4 & 1.1 & 0.01 & 0.27 & -0.32 & 0.16 & 0.14 & 0.11 & 0.26 \\
\hline 198 & 4384 & 31 & 0.7 & 0.9 & 0.9 & 1.07 & 0.02 & 0.37 & -0.53 & 0.18 & 0.15 & 0.0 & 0.29 \\
\hline 206 & 4586 & 31 & 1.0 & 1.1 & 1.2 & 1.07 & 0.02 & 0.5 & -1.1 & -0.49 & 0.16 & 0.94 & 0.29 \\
\hline 208 & 4392 & 30 & 0.8 & 0.9 & 1.0 & 1.09 & 0.02 & 0.28 & -0.68 & -0.01 & 0.14 & 0.19 & 0.26 \\
\hline 210 & 4458 & 30 & 0.8 & 1.0 & 1.0 & 1.07 & 0.01 & 0.38 & -0.75 & -0.06 & .15 & 0.48 & 0.27 \\
\hline 220 & 4575 & 31 & 1.0 & 1.2 & 1.2 & 1.08 & 0.01 & 0.43 & -1.1 & -0.54 & 0.15 & 1.06 & 0.28 \\
\hline 10001 & 4588 & 31 & 1.0 & 1.1 & 1.2 & 1.07 & 0.02 & 0.44 & -0.45 & 0.22 & 0.15 & 0.16 & 0.29 \\
\hline 10002 & 4406 & 31 & 0.8 & 1.0 & 1.0 & 1.09 & 0.02 & 0.31 & -0.66 & 0.01 & 0.14 & -0.28 & 0.27 \\
\hline 10003 & 4842 & 32 & 1.5 & 1.6 & 1.7 & 1.11 & 0.01 & 0.5 & -1.15 & -0.77 & 0.18 & 0.73 & 0.33 \\
\hline 10004 & 4807 & 34 & 1.5 & 1.7 & 1.8 & 1.11 & 0.01 & 0.41 & -0.54 & -0.21 & 0.15 & 0.77 & 0.3 \\
\hline 20001 & 4601 & 31 & 1.1 & 1.2 & 1.2 & 1.08 & 0.01 & 0.45 & -0.38 & 0.25 & 0.15 & 0.23 & 0.27 \\
\hline 20002 & 4631 & 66 & 1.1 & 1.2 & 1.4 & 1.08 & 0.02 & 0.5 & 1.3 & 1.49 & 0.17 & 0.87 & 0.31 \\
\hline 20003 & 4852 & 35 & 1.5 & 1.6 & 1.7 & 1.11 & 0.01 & 0.5 & 0.12 & 0.49 & 0.18 & -0.22 & u.l. \\
\hline 20005 & 4676 & 31 & 1.2 & 1.3 & 1.4 & 1.08 & 0.01 & 0.48 & -1.03 & -0.38 & 0.17 & -0.52 & u.l. \\
\hline 30001 & 4653 & 31 & 1.4 & 1.5 & 1.6 & 1.11 & 0.01 & 0.19 & -1.01 & -0.55 & 0.13 & 0.3 & 0.26 \\
\hline 30002 & 4865 & 32 & 1.7 & 1.9 & 1.9 & 1.13 & 0.01 & 0.31 & -0.09 & 0.04 & 0.15 & 0.42 & 0.33 \\
\hline 50001 & 4607 & 31 & 1.2 & 1.4 & 1.5 & 1.11 & 0.01 & 0.15 & -0.29 & 0.13 & 0.13 & 0.4 & 0.35 \\
\hline 50002 & 4499 & 31 & 0.8 & 0.8 & 0.9 & 1.04 & 0.01 & 0.5 & -1.05 & 0.43 & 0.19 & 0.78 & 0.35 \\
\hline 50004 & 4704 & 65 & 1.2 & 1.4 & 1.6 & 1.09 & 0.02 & 0.46 & -0.39 & 0.14 & 0.17 & 0.47 & 0.32 \\
\hline 90015 & 4292 & 30 & 0.8 & 0.8 & 1.0 & 1.11 & 0.02 & 0.04 & -0.9 & -0.2 & 0.12 & 0.01 & 0.24 \\
\hline 90018 & 4843 & 33 & 1.5 & 1.6 & 1.7 & 1.1 & 0.01 & 0.5 & -0.66 & -0.3 & 0.24 & 0.46 & u.l. \\
\hline 90026 & 4507 & 31 & 1.1 & 1.2 & 1.4 & 1.1 & 0.02 & 0.13 & -0.69 & -0.16 & 0.12 & 0.07 & 0.23 \\
\hline 90027 & 4235 & 30 & 0.8 & 0.9 & 1.0 & 1.14 & 0.02 & -0.07 & -0.84 & -0.34 & 0.11 & -0.01 & 0.23 \\
\hline 90029 & 4458 & 31 & 1.0 & 1.1 & 1.2 & 1.1 & 0.02 & 0.11 & -0.58 & -0.03 & 0.12 & 0.18 & 0.23 \\
\hline 90030 & 4351 & 31 & 1.0 & 1.1 & 1.3 & 1.14 & 0.02 & -0.13 & -0.75 & -0.31 & 0.24 & 0.03 & 0.35 \\
\hline 90042 & 4640 & 32 & 1.4 & 1.5 & 1.6 & 1.12 & 0.01 & 0.12 & -0.41 & 0.01 & 0.13 & -0.05 & u.1. \\
\hline 90045 & 4543 & 31 & 1.2 & 1.4 & 1.5 & 1.13 & 0.02 & 0.07 & -0.46 & -0.01 & 0.12 & -0.07 & 0.22 \\
\hline 90050 & 4762 & 32 & 1.3 & 1.4 & 1.5 & 1.09 & 0.01 & 0.5 & -0.84 & -0.28 & 0.18 & -0.09 & u.1. \\
\hline 90057 & 4980 & 37 & 1.9 & 2.0 & 2.1 & 1.14 & 0.01 & 0.39 & -0.63 & -0.56 & 0.16 & 0.72 & 0.32 \\
\hline 90069 & 4508 & 31 & 1.1 & 1.1 & 1.3 & 1.09 & 0.01 & 0.21 & -0.82 & -0.13 & 0.13 & 0.17 & 0.25 \\
\hline 90070 & 4609 & 31 & 1.3 & 1.4 & 1.5 & 1.11 & 0.01 & 0.16 & -0.76 & -0.23 & 0.13 & 0.27 & 0.23 \\
\hline 90074 & 4945 & 33 & 1.8 & 2.0 & 2.1 & 1.14 & 0.01 & 0.37 & -0.47 & -0.4 & 0.16 & 1.0 & 0.31 \\
\hline
\end{tabular}


Table A.2. continued.

\begin{tabular}{|c|c|c|c|c|c|c|c|c|c|c|c|c|c|}
\hline ID & $\begin{array}{r}T \\
(\mathrm{~K})\end{array}$ & $\begin{array}{l}e T \\
(\mathrm{~K})\end{array}$ & $\begin{array}{r}\log (g)-l \\
(\text { dex })\end{array}$ & $\begin{array}{r}\log (g) \\
(\text { dex })\end{array}$ & $\begin{array}{r}\log (g)-u \\
(\text { dex })\end{array}$ & $\begin{array}{r}v_{\mathrm{t}} \\
\left(\mathrm{km} \mathrm{s}^{-1}\right)\end{array}$ & $\begin{array}{r}e v_{\mathrm{t}} \\
\left(\mathrm{km} \mathrm{s}^{-1}\right)\end{array}$ & $\begin{array}{r}{[\mathrm{O} / \mathrm{Fe}]} \\
(\mathrm{dex})\end{array}$ & $\begin{array}{r}{[\mathrm{C} / \mathrm{Fe}]} \\
(\mathrm{dex})\end{array}$ & $\begin{array}{r}{[\mathrm{C} / \mathrm{Fe}]_{\text {cor }}} \\
(\mathrm{dex})\end{array}$ & $\begin{array}{r}e[\mathrm{C} / \mathrm{Fe}] \\
(\mathrm{dex})\end{array}$ & $\begin{array}{r}{[N / \mathrm{Fe}]} \\
(\mathrm{dex})\end{array}$ & $\begin{array}{r}e[N / \mathrm{Fe}] \\
(\mathrm{dex})\end{array}$ \\
\hline$\overline{90085}$ & 5073 & 35 & 2.1 & 2.2 & 2.3 & 1.16 & 0.01 & 0.5 & 1.35 & 1.38 & 0.15 & 1.59 & 0.25 \\
\hline 90094 & 4815 & 34 & 1.5 & 1.5 & 1.6 & 1.1 & 0.01 & 0.5 & -0.07 & 0.36 & 0.2 & 1.01 & 0.35 \\
\hline 90096 & 4530 & 31 & 0.8 & 0.9 & 1.0 & 1.04 & 0.01 & 0.5 & -0.71 & 0.06 & 0.18 & 0.56 & 0.35 \\
\hline 90104 & 4263 & 30 & 0.6 & 0.6 & 0.8 & 1.08 & 0.02 & 0.23 & -0.9 & 0.17 & 0.13 & 0.03 & 0.28 \\
\hline 90109 & 4939 & 35 & 1.8 & 1.9 & 2.1 & 1.13 & 0.01 & 0.44 & -0.87 & -0.74 & 0.16 & 0.3 & u.1. \\
\hline 90112 & 4022 & 30 & 0.5 & 0.6 & 0.6 & 1.17 & 0.02 & 0.12 & -1.02 & -0.26 & 0.16 & -0.35 & u.l. \\
\hline 90118 & 4068 & 30 & 0.5 & 0.5 & 0.6 & 1.14 & 0.02 & 0.33 & -0.68 & -0.01 & 0.15 & 0.45 & 0.27 \\
\hline 90121 & 4446 & 31 & 0.9 & 1.0 & 1.1 & 1.08 & 0.02 & 0.2 & -1.23 & -0.54 & 0.13 & 0.28 & 0.25 \\
\hline 90122 & 4462 & 31 & 1.2 & 1.3 & 1.4 & 1.13 & 0.01 & -0.09 & -1.18 & -0.6 & 0.11 & -0.22 & 0.23 \\
\hline 123 & 4353 & 31 & 0.8 & 0.8 & 0.9 & 1.08 & 0.02 & 0.24 & -1.14 & -0.4 & 0.13 & 0.11 & 0.26 \\
\hline 90130 & 4448 & 31 & 0.9 & 1.0 & 1.1 & 1.08 & 0.02 & 0.2 & -0.83 & -0.21 & 0.13 & 0.04 & 0.25 \\
\hline 90131 & 4093 & 30 & 0.6 & 0.6 & 0.6 & 1.13 & 0.02 & 0.19 & -1.01 & -0.28 & 0.13 & -0.23 & 0.3 \\
\hline 90325 & 4826 & 31 & 1.7 & 1.8 & 2.0 & 1.13 & 0.01 & 0.11 & -1.0 & -0.7 & 0.13 & 0.12 & 0.28 \\
\hline 90343 & 4490 & 31 & 1.0 & 1.1 & 1.2 & 1.08 & 0.02 & 0.24 & -0.97 & -0.27 & 0.13 & 0.17 & 0.25 \\
\hline 90344 & 4622 & 65 & 1.3 & 1.3 & 1.5 & 1.09 & 0.02 & 0.22 & -0.28 & 0.21 & 0.16 & 0.1 & 0.3 \\
\hline 90354 & 4709 & 65 & 1.4 & 1.6 & 1.7 & 1.12 & 0.02 & 0.2 & -0.55 & -0.2 & 0.16 & -0.26 & 0.3 \\
\hline 90355 & 4861 & 37 & 1.7 & 1.8 & 2.0 & 1.13 & 0.01 & 0.21 & -0.46 & -0.22 & 0.14 & 0.01 & u.1. \\
\hline 90361 & 4632 & 31 & 1.3 & 1.4 & 1.5 & 1.11 & 0.01 & 0.12 & -1.17 & -0.61 & 0.13 & 0.03 & 0.23 \\
\hline 90365 & 4900 & 36 & 1.7 & 1.9 & 2.0 & 1.13 & 0.01 & 0.37 & -0.1 & 0.01 & 0.15 & -0.31 & 0.3 \\
\hline 90368 & 4621 & 31 & 1.3 & 1.3 & 1.5 & 1.09 & 0.02 & 0.23 & -0.42 & 0.1 & 0.13 & 0.13 & 0.23 \\
\hline 90448 & 4437 & 31 & 1.0 & 1.1 & 1.3 & 1.1 & 0.02 & 0.11 & -0.76 & -0.21 & 0.12 & -0.09 & 0.23 \\
\hline 90449 & 4929 & 34 & 1.8 & 1.9 & 2.0 & 1.14 & 0.01 & 0.33 & -0.76 & -0.61 & 0.15 & 0.22 & 0.31 \\
\hline 90452 & 4179 & 30 & 0.5 & 0.6 & 0.6 & 1.1 & 0.02 & 0.3 & -0.87 & -0.12 & 0.14 & -0.13 & 0.3 \\
\hline 90455 & 4683 & 32 & 1.4 & 1.4 & 1.6 & 1.1 & 0.01 & 0.24 & -0.6 & -0.11 & 0.14 & 0.08 & 0.25 \\
\hline 90457 & 4139 & 30 & 0.5 & 0.5 & 0.6 & 1.1 & 0.01 & 0.33 & -0.87 & -0.13 & 0.14 & 0.45 & 0.32 \\
\hline 90458 & 4089 & 30 & 0.5 & 0.5 & 0.5 & 1.13 & 0.02 & 0.22 & -1.06 & -0.33 & 0.14 & 0.15 & 0.31 \\
\hline 90549 & 4435 & 31 & 1.0 & 1.0 & 1.1 & 1.1 & 0.01 & 0.16 & -0.94 & -0.23 & 0.13 & 0.15 & 0.24 \\
\hline 90552 & 3906 & 30 & 0.4 & 0.4 & 0.5 & 1.2 & 0.02 & 0.08 & -0.85 & -0.28 & 0.13 & 0.07 & 0.3 \\
\hline 90553 & 4032 & 30 & 0.4 & 0.4 & 0.5 & 1.14 & 0.02 & 0.0 & -1.13 & 0.0 & 0.12 & 0.05 & 0.27 \\
\hline
\end{tabular}

